\title{
Existence results for a functional boundary value problem of fractional differential equations
}

\author{
Yumei Zou ${ }^{1}$ and Yujun Cui ${ }^{2 *}$
}

\section{*Correspondence:}

cyj720201@163.com

2Department of Mathematics,

Shandong University of Science and

Technology, Qingdao, 266590,

P.R. China

Full list of author information is

available at the end of the article

\begin{abstract}
In this paper, a functional boundary value problem of fractional differential equations is studied. Based on Mawhin's coincidence degree theory, some existence theorems are obtained in the case of nonresonance and the cases of $\operatorname{dim} \operatorname{Ker} L=1$ and $\operatorname{dim} \operatorname{Ker} L=2$ at resonance.
\end{abstract}

\section{Introduction}

The subject of fractional calculus has gained considerable popularity and importance because of its frequent appearance in various fields such as physics, chemistry, and engineering. In consequence, the subject of fractional differential equations has attracted much attention. Many methods have been introduced to solve fractional differential equations, such as the popular Laplace transform method, the iteration method, the Fourier transform method and the operational method. For details, see [1-3] and the references therein. Recently, there have been some papers dealing with the basic theory for initial value problems of nonlinear fractional differential equations; for example, see [4, 5]. Also, there are some articles which deal with the existence and multiplicity of solutions for nonlinear boundary value problems of fractional order differential equations using techniques of topological degree theory. We refer the reader to [6-16] for some recent results at nonresonance and to [17-26] at resonance.

In [18], by making use of the coincidence degree theory of Mawhin, Zhang and Bai discussed the existence results for the following nonlinear nonlocal problem at resonance under the case $\operatorname{dim} \operatorname{Ker} L=1$ :

$$
D_{0+}^{\alpha} u(t)=f(t, u(t)), \quad 0<t<1, \quad u(0)=0, \quad \beta u(\eta)=u(1), \quad 1<\alpha \leq 2 .
$$

Recently, Jiang [26] studied the existence of a solution for the following fractional differential equation at resonance under the case $\operatorname{dim} \operatorname{Ker} L=2$ :

$$
\begin{aligned}
& D_{0+}^{\alpha} u(t)=f\left(t, u(t), D_{0+}^{\alpha-1} u(t)\right), \\
& u(0)=0, \quad D_{0+}^{\alpha-1} u(0)=\sum_{i=1}^{m} a_{i} D_{0+}^{\alpha-1} u\left(\xi_{i}\right), \quad D_{0+}^{\alpha-2} u(1)=\sum_{j=1}^{n} b_{j} D_{0+}^{\alpha-2} u\left(\eta_{j}\right) .
\end{aligned}
$$


Being directly inspired by $[18,20,26]$, we intend in this paper to study the following functional boundary value problems (FBVP) of fractional order differential equation:

$$
\begin{aligned}
& D_{0+}^{\alpha} u(t)=f\left(t, u(t), D_{0+}^{\alpha-1} u(t), D_{0+}^{\alpha-2} u(t)\right), \\
& \left.I_{0+}^{3-\alpha} u(t)\right|_{t=0}=0, \quad \Phi_{1}\left[D_{0+}^{\alpha-1} u(t)\right]=0, \quad \Phi_{2}\left[D_{0+}^{\alpha-2} u(t)\right]=0,
\end{aligned}
$$

where $2<\alpha<3, D_{0_{+}}^{\alpha}$ and $I_{0+}^{\alpha}$ are the standard Riemann-Liouville differentiation and integration, and $f \in C\left([0,1] \times \mathbb{R}^{3}, \mathbb{R}\right) ; \Phi_{1}, \Phi_{2}: C[0,1] \rightarrow \mathbb{R}$ are continuous linear functionals.

In this paper, we shall give some sufficient conditions to construct the existence theorems for FBVP (1.1), (1.2) at nonresonance and resonance (both cases of $\operatorname{dim} \operatorname{Ker} L=1$ and $\operatorname{dim} \operatorname{Ker} L=2$ ), respectively. To the best of our knowledge, the method of Mawhin's theorem has not been developed for fractional order differential equation with functional boundary value problems at resonance. So, it is interesting and important to discuss the existence of a solution for FBVP (1.1), (1.2). Many difficulties occur when we deal with them. For example, the construction of the generalized inverse $K_{p}: \operatorname{Im} L \rightarrow \operatorname{dom} L \cap \operatorname{Ker} P$ of $L$. So, we need to introduce some new tools and methods to investigate the existence of a solution for FBVP (1.1), (1.2).

The rest of this paper is organized as follows. In Section 2, we give some notations and lemmas. In Section 3, we establish the existence results of a solution for functional boundary value problem (1.1), (1.2).

\section{Preliminaries and lemmas}

For the convenience of the reader, we present here the necessary definitions from fractional calculus theory. These definitions and properties can be found in the literature. The readers who are unfamiliar with this area can consult, for example, $[1,2,4]$ for details.

Definition 2.1 [1,2] The Riemann-Liouville fractional integral of order $\alpha>0$ of a function $u:(0, \infty) \rightarrow \mathbb{R}$ is given by

$$
I_{0+}^{\alpha} u(t)=\frac{1}{\Gamma(\alpha)} \int_{0}^{t}(t-s)^{\alpha-1} u(s) d s,
$$

provided that the right-hand side is pointwise defined on $(0, \infty)$. Here $\Gamma(\alpha)$ is the Gamma function given by $\Gamma(\alpha)=\int_{0}^{+\infty} t^{\alpha-1} e^{-t} d t$.

Definition 2.2 [1,2] The Riemann-Liouville fractional derivative of order $\alpha>0$ of a continuous function $u:(0, \infty) \rightarrow \mathbb{R}$ is given by

$$
D_{0^{+}}^{\alpha} u(t)=\frac{1}{\Gamma(n-\alpha)}\left(\frac{d}{d t}\right)^{n} \int_{0}^{t} \frac{u(s)}{(t-s)^{\alpha-n+1}} d s,
$$

where $n-1 \leq \alpha<n$, provided that the right-hand side is pointwise defined on $(0, \infty)$.

We use the classical spaces $C[0,1]$ with the norm $\|u\|_{\infty}=\max _{t \in[0,1]}|u(t)|, L^{1}[0,1]$ with the norm $\|u\|_{1}=\int_{0}^{1}|u(t)| d t$. We also use the space $A C^{n}[0,1]$ defined by

$$
A C^{n}[0,1]=\left\{u:[0,1] \rightarrow \mathbb{R} \mid u^{(n-1)} \text { are absolutely continuous on }[0,1]\right\}
$$


and the Banach space $C^{\mu}[0,1](\mu>0)$

$$
\begin{aligned}
C^{\mu}[0,1]= & \left\{u(t) \mid u(t)=I_{0_{+}}^{\mu} x(t)+c_{1} t^{\mu-1}+c_{2} t^{\mu-2}+\cdots+c_{N-1} t^{\mu-(N-1)},\right. \\
& \left.x \in C[0,1], t \in[0,1], c_{i} \in \mathbb{R}, i=1,2, \ldots, N=[\mu]+1\right\}
\end{aligned}
$$

with the norm $\|u\|_{C^{\mu}}=\left\|D_{0_{+}}^{\mu} u\right\|_{\infty}+\cdots+\left\|D_{0+}^{\mu-(N-1)} u\right\|_{\infty}+\|u\|_{\infty}$.

Lemma 2.1 [2] Let $\alpha>0, n=[\alpha]+1$. Assume that $u \in L^{1}(0,1)$ with a fractional integration of order $n-\alpha$ that belongs to $A C^{n}[0,1]$. Then the equality

$$
\left(I_{0+}^{\alpha} D_{0+}^{\alpha} u\right)(t)=u(t)-\sum_{i=1}^{n} \frac{\left.\left(\left(I_{0+}^{n-\alpha} u\right)(t)\right)^{(n-i)}\right|_{t=0}}{\Gamma(\alpha-i+1)} t^{\alpha-i}
$$

holds almost everywhere on $[0,1]$.

Remark 2.1 If $u$ satisfies $D_{0+}^{\alpha} u=f(t) \in L^{1}(0,1)$ and $\left.I_{0+}^{3-\alpha} u\right|_{t=0}=0$, then $u \in C^{\alpha-1}[0,1]$. In fact, with Lemma 2.1, one has

$$
u(t)=I_{0+}^{\alpha} f(t)+c_{1} t^{\alpha-1}+c_{2} t^{\alpha-2}+c_{3} t^{\alpha-3} .
$$

Combine with $\left.I_{0+}^{3-\alpha} u\right|_{t=0}=0$, there is $c_{3}=0$. So,

$$
u(t)=I_{0+}^{\alpha} f(t)+c_{1} t^{\alpha-1}+c_{2} t^{\alpha-2}=I_{0+}^{\alpha-1}\left[I_{0+}^{1} f(t)+c_{1} \Gamma(\alpha)\right]+c_{2} t^{(\alpha-1)-1} .
$$

In the following lemma, we use the unified notation both for fractional integrals and fractional derivatives assuming that $I_{0_{+}}^{\alpha}=D_{0_{+}}^{-\alpha}$ for $\alpha<0$.

Lemma 2.2 [2] Assume $\alpha>0$, then:

(i) Let $k \in \mathbb{N}$. If $D_{a+}^{\alpha} u(t)$ and $\left(D_{a+}^{\alpha+k} u\right)(t)$ exist, then

$$
\left(D^{k} D_{a+}^{\alpha}\right) u(t)=\left(D_{a+}^{\alpha+k} u\right)(t)
$$

(ii) If $\beta>0, \alpha+\beta>1$, then

$$
\left(I_{a+}^{\alpha} I_{a+}^{\beta}\right) u(t)=\left(I_{a+}^{\alpha+\beta} u\right)(t)
$$

is satisfied at any point on $[a, b]$ for $u \in L_{p}(a, b)$ and $1 \leq p \leq+\infty$;

(iii) Let $u \in C[a, b]$. Then $\left(D_{a+}^{\alpha} I_{a+}^{\alpha}\right) u(t)=u(t)$ holds on $[a, b]$;

(iv) Note that for $\lambda>-1, \lambda \neq \alpha-1, \alpha-2, \ldots, \alpha-n$, we have

$$
D^{\alpha} t^{\lambda}=\frac{\Gamma(\lambda+1)}{\Gamma(\lambda-\alpha+1)} t^{\lambda-\alpha}, \quad D^{\alpha} t^{\alpha-i}=0, \quad i=1,2, \ldots, n .
$$

Lemma 2.3 [18] $F \subset C^{\mu}[0,1]$ is a sequentially compact set if and only if $F$ is uniformly bounded and equicontinuous. Here ' $F$ is uniformly bounded and equicontinuous' means that there exists $M>0$ such that for every $u \in F$,

$$
\|u\|_{C^{\mu}}=\left\|D_{0_{+}}^{\mu} u\right\|_{\infty}+\cdots+\left\|D_{0+}^{\mu-[\mu]} u\right\|_{\infty}+\|u\|_{\infty}<M
$$


and that $\forall \epsilon>0, \exists N>0$, for all $t_{1}, t_{2} \in[0,1],\left|t_{1}-t_{2}\right|<\delta, u \in F, i \in\{0,1, \ldots,[\mu]\}$, there hold

$$
\left|u\left(t_{1}\right)-u\left(t_{2}\right)\right|<\varepsilon, \quad\left|D_{0+}^{\mu-i} u\left(t_{1}\right)-D_{0+}^{\mu-i} u\left(t_{2}\right)\right|<\varepsilon,
$$

respectively.

Next, consider the following conditions:

(A1) $\Phi_{1}[1] \Phi_{2}[1] \neq 0$.

(A2) $\Phi_{1}[1]=0, \Phi_{2}[1] \neq 0, \Phi_{2}[t]=0$.

(A3) $\Phi_{1}[1]=0, \Phi_{2}[1]=0, \Phi_{2}[t] \neq 0$.

(A4) $\Phi_{1}[1] \neq 0, \Phi_{2}[1]=0, \Phi_{2}[t]=0$.

(A5) $\Phi_{1}[1]=0, \Phi_{2}[1]=0, \Phi_{2}[t]=0$.

We shall prove that: If (A1) holds, then $\operatorname{Ker} L=\{\theta\}$. It is the so-called nonresonance case. If (A2) holds, then $\operatorname{Ker} L=\left\{a t^{\alpha-1}: a \in \mathbb{R}\right\}$. If (A3) or (A4) holds, then $\operatorname{Ker} L=\left\{a t^{\alpha-2}: a \in \mathbb{R}\right\}$. If (A5) holds, then $\operatorname{Ker} L=\left\{a t^{\alpha-1}+b t^{\alpha-2}: a, b \in \mathbb{R}\right\}$.

In the nonresonance case, FBVP (1.1), (1.2) can be transformed into an operator equation.

Lemma 2.4 Assume that (A1) holds. Then functional boundary value problem (1.1) and (1.2) has a solution if and only if the operator $T: C^{\alpha-1}[0,1] \rightarrow C^{\alpha-1}[0,1]$, defined by

$$
\begin{aligned}
(T u)(t)= & \frac{1}{\Gamma(\alpha)} \int_{0}^{t}(t-s)^{\alpha-1}(\mathbf{f} u)(s) d s-\frac{\Phi_{1}\left[\int_{0}^{t}(\mathbf{f} u)(s) d s\right]}{\Gamma(\alpha) \Phi_{1}[1]} t^{\alpha-1} \\
& -\frac{\Phi_{1}[1] \Phi_{2}\left[\int_{0}^{t}(t-s)(\mathbf{f} u)(s) d s\right]-\Phi_{1}\left[\int_{0}^{t}(\mathbf{f} u)(s) d s\right] \Phi_{2}[t]}{\Gamma(\alpha-1) \Phi_{1}[1] \Phi_{2}[1]} t^{\alpha-2},
\end{aligned}
$$

has a fixed point, where $(\mathbf{f} u)(t)=f\left(t, u(t), D_{0+}^{\alpha-1} u(t), D_{0+}^{\alpha-2} u(t)\right)$.

Proof If $u$ is a solution to $T u=u$, by Lemma 2.2, we get

$$
\begin{aligned}
& D_{0+}^{\alpha} u(t)=f\left(t, u(t), D_{0+}^{\alpha-1} u(t), D_{0+}^{\alpha-2} u(t)\right), \\
& D_{0+}^{\alpha-1} u(t)=\int_{0}^{t}(\mathbf{f} u)(s) d s-\frac{\Phi_{1}\left[\int_{0}^{t}(\mathbf{f} u)(s) d s\right]}{\Phi_{1}[1]}
\end{aligned}
$$

and

$$
\begin{aligned}
D_{0+}^{\alpha-2} u(t)= & \int_{0}^{t}(t-s)(\mathbf{f} u)(s) d s-\frac{\Phi_{1}\left[\int_{0}^{t}(\mathbf{f} u)(s) d s\right]}{\Phi_{1}[1]} t \\
& -\frac{\Phi_{1}[1] \Phi_{2}\left[\int_{0}^{t}(t-s)(\mathbf{f} u)(s) d s\right]-\Phi_{1}\left[\int_{0}^{t}(\mathbf{f} u)(s) d s\right] \Phi_{2}[t]}{\Phi_{1}[1] \Phi_{2}[1]}
\end{aligned}
$$

Considering the linearity of $\Phi_{i}(i=1,2)$, we have

$$
\begin{aligned}
& \left.I_{0+}^{3-\alpha} u(t)\right|_{t=0}=0 \\
& \Phi_{1}\left[D_{0+}^{\alpha-1} u(t)\right]=\Phi_{1}\left[\int_{0}^{t}(\mathbf{f} u)(s) d s\right]-\frac{\Phi_{1}\left[\int_{0}^{t}(\mathbf{f} u)(s) d s\right]}{\Phi_{1}[1]} \Phi_{1}[1]=0,
\end{aligned}
$$




$$
\begin{aligned}
\Phi_{2}\left[D_{0+}^{\alpha-2} u(t)\right]= & \Phi_{2}\left[\int_{0}^{t}(t-s)(\mathbf{f} u)(s) d s\right]-\frac{\Phi_{1}\left[\int_{0}^{t}(\mathbf{f} u)(s) d s\right]}{\Phi_{1}[1]} \Phi_{2}[t] \\
& -\frac{\Phi_{1}[1] \Phi_{2}\left[\int_{0}^{t}(t-s)(\mathbf{f} u)(s) d s\right]-\Phi_{1}\left[\int_{0}^{t}(\mathbf{f} u)(s) d s\right] \Phi_{2}[t]}{\Phi_{1}[1] \Phi_{2}[1]} \Phi_{2}[1]=0 .
\end{aligned}
$$

So, $u$ is a solution to FBVP (1.1), (1.2).

If $u$ is a solution to (1.1), by Lemma 2.1, we can reduce (1.1) to an equivalent integral equation

$$
u(t)=I_{0+}^{\alpha}(\mathbf{f} u)(t)+c_{1} t^{\alpha-1}+c_{2} t^{\alpha-2}+c_{3} t^{\alpha-3} .
$$

By $\left.I_{0+}^{3-\alpha} u(t)\right|_{t=0}=0$, there is $c_{3}=0$, and

$$
\begin{aligned}
& D_{0+}^{\alpha-1} u(t)=\int_{0}^{t}(\mathbf{f} u)(s) d s+c_{1} \Gamma(\alpha), \\
& D_{0+}^{\alpha-2} u(t)=\int_{0}^{t}(t-s)(\mathbf{f} u)(s) d s+c_{1} \Gamma(\alpha) t+c_{2} \Gamma(\alpha-1) .
\end{aligned}
$$

Applying $\Phi_{1}$ and $\Phi_{2}$ to (2.2) and (2.3), respectively, we obtain

$$
\begin{aligned}
& 0=\Phi_{1}\left[D_{0+}^{\alpha-1} u(t)\right]=\Phi_{1}\left[\int_{0}^{t}(\mathbf{f} u)(s) d s\right]+c_{1} \Gamma(\alpha) \Phi_{1}[1] \\
& 0=\Phi_{2}\left[D_{0+}^{\alpha-2} u(t)\right]=\Phi_{2}\left[\int_{0}^{t}(t-s)(\mathbf{f} u)(s) d s\right]+c_{1} \Gamma(\alpha) \Phi_{2}[t]+c_{2} \Gamma(\alpha-1) \Phi_{2}[1] .
\end{aligned}
$$

Thus,

$$
\begin{aligned}
& c_{1}=-\frac{\Phi_{1}\left[\int_{0}^{t}(\mathbf{f} u)(s) d s\right]}{\Gamma(\alpha) \Phi_{1}[1]}, \\
& c_{2}=-\frac{\Phi_{1}[1] \Phi_{2}\left[\int_{0}^{t}(t-s)(\mathbf{f} u)(s) d s\right]-\Phi_{1}\left[\int_{0}^{t}(\mathbf{f} u)(s) d s\right] \Phi_{2}[t]}{\Gamma(\alpha-1) \Phi_{1}[1] \Phi_{2}[1]} .
\end{aligned}
$$

Substituting (2.4) and (2.5) into (2.1), we obtain

$$
\begin{aligned}
u(t)= & \frac{1}{\Gamma(\alpha)} \int_{0}^{t}(t-s)^{\alpha-1}(\mathbf{f} u)(s) d s-\frac{\Phi_{1}\left[\int_{0}^{t}(\mathbf{f} u)(s) d s\right]}{\Gamma(\alpha) \Phi_{1}[1]} t^{\alpha-1} \\
& -\frac{\Phi_{1}[1] \Phi_{2}\left[\int_{0}^{t}(t-s)(\mathbf{f} u)(s) d s\right]-\Phi_{1}\left[\int_{0}^{t}(\mathbf{f} u)(s) d s\right] \Phi_{2}[t]}{\Gamma(\alpha-1) \Phi_{1}[1] \Phi_{2}[1]} t^{\alpha-2} .
\end{aligned}
$$

The proof is complete.

The following definitions and lemmas are a preparation for the existence of solutions to (1.1), (1.2) at resonance.

Definition 2.3 Let $Y, Z$ be real Banach spaces, let $L: \operatorname{dom} L \subset Y \rightarrow Z$ be a linear operator. $L$ is said to be a Fredholm operator of index zero provided that:

(i) $\operatorname{Im} L$ is a closed subset of $Z$,

(ii) $\operatorname{dim} \operatorname{Ker} L=\operatorname{codim} \operatorname{Im} L<+\infty$. 
Let $Y, Z$ be real Banach spaces and $L: \operatorname{dom} L \subset Y \rightarrow Z$ be a Fredholm operator of index zero. $P: Y \rightarrow Y, Q: Z \rightarrow Z$ are continuous projectors such that $\operatorname{Im} P=\operatorname{Ker} L, \operatorname{Ker} Q=\operatorname{Im} L$, $Y=\operatorname{Ker} L \oplus \operatorname{Ker} P$ and $Z=\operatorname{Im} L \oplus \operatorname{Im} Q$. It follows that $\left.L\right|_{\operatorname{dom} L \cap \operatorname{Ker} P}: \operatorname{dom} L \cap \operatorname{Ker} P \rightarrow \operatorname{Im} L$ is invertible. We denote the inverse of the mapping by $K_{P}$ (generalized inverse operator of $L$ ). If $\Omega$ is an open bounded subset of $Y$ such that $\operatorname{dom} L \cap \Omega \neq \emptyset$, the mapping $N: Y \rightarrow Z$ will be called $L$-compact on $\bar{\Omega}$, if $Q N(\bar{\Omega})$ is bounded and $K_{P}(I-Q) N: \bar{\Omega} \rightarrow Y$ is compact.

We need the following known result for the sequel (Theorem 2.4 [27]).

Theorem 2.1 Let $L$ be a Fredholm operator of index zero, and let $N$ be L-compact on $\bar{\Omega}$. Assume that the following conditions are satisfied:

(i) $L x \neq \lambda N x$ for every $(x, \lambda) \in[(\operatorname{dom} L \backslash \operatorname{Ker} L) \cap \partial \Omega] \times(0,1)$.

(ii) $N x \notin \operatorname{Im} L$ for every $x \in \operatorname{Ker} L \cap \partial \Omega$.

(iii) $\operatorname{deg}\left(\left.Q N\right|_{\operatorname{Ker} L}, \operatorname{Ker} L \cap \Omega, 0\right) \neq 0$, where $Q: Z \rightarrow Z$ is a projector as above with $\operatorname{Im} L=\operatorname{Ker} Q$.

Then the equation $L x=N x$ has at least one solution in $\operatorname{dom} L \cap \bar{\Omega}$.

Let $Y=C^{\alpha-1}[0,1], Z=L^{1}[0,1]$. Let the linear operator $L: Y \subset \operatorname{dom} L \rightarrow Z$ with

$$
\begin{aligned}
\operatorname{dom} L= & \left\{u \in C^{\alpha-1}[0,1]: D_{0+}^{\alpha} u(t) \in Z,\left.I_{0+}^{3-\alpha} u(t)\right|_{t=0}=0\right. \\
& \left.\Phi_{1}\left[D_{0+}^{\alpha-1} u(t)\right]=0, \Phi_{2}\left[D_{0+}^{\alpha-2} u(t)\right]=0\right\}
\end{aligned}
$$

be defined by $L u=D_{0_{+}}^{\alpha} u(t)$. Let the nonlinear operator $N: Y \rightarrow Z$ be defined by

$$
(N u)(t)=f\left(t, u(t), D_{0+}^{\alpha-1} u(t), D_{0+}^{\alpha-2} u(t)\right)
$$

Then (1.1), (1.2) can be written as

$$
L u=N u \text {. }
$$

Now, we give $\operatorname{Ker} L, \operatorname{Im} L$ and some necessary operators at $\operatorname{dim} \operatorname{Ker} L=1$ and $\operatorname{dim} \operatorname{Ker} L=$ 2 , respectively.

Lemma 2.5 Let $L$ be the linear operator defined as above. If (A2) holds, then

$$
\operatorname{Ker} L=\left\{u \in \operatorname{dom} L: u=a t^{\alpha-1}, a \in \mathbb{R}, t \in[0,1]\right\}
$$

and

$$
\operatorname{Im} L=\left\{v \in Z: \Phi_{1}\left[\int_{0}^{t} v(s) d s\right]=0\right\} .
$$

Proof Let $u(t)=a t^{\alpha-1}$. Clearly, $D_{0+}^{\alpha} u(t)=0$ and $\left.I_{0+}^{3-\alpha} u(t)\right|_{t=0}=0$. Considering (A2), $\Phi_{1}\left[D_{0+}^{\alpha-1} u(t)\right]=a \Phi_{1}[\Gamma(\alpha)]=a \Gamma(\alpha) \Phi_{1}[1]=0$ and $\Phi_{2}\left[D_{0+}^{\alpha-2} u(t)\right]=\Gamma(\alpha) \Phi_{2}[t]=0$. So,

$$
\left\{u \in \operatorname{dom} L: u=a t^{\alpha-1}, a \in \mathbb{R}, t \in[0,1]\right\} \subset \operatorname{Ker} L .
$$


If $L u=D_{0+}^{\alpha} u(t)=0$, then $u(t)=a t^{\alpha-1}+b t^{\alpha-2}+c t^{\alpha-3}$. Considering $\left.I_{0+}^{3-\alpha} u(t)\right|_{t=0}=0$ and (A2), we can obtain that $b=c=0$. It yields $u(t)=a t^{\alpha-1}$ and $\operatorname{Ker} L \subset\left\{u \in \operatorname{dom} L: u=a t^{\alpha-1}, a \in\right.$ $\mathbb{R}, t \in[0,1]\}$.

We now show that

$$
\operatorname{Im} L=\left\{v \in Z: \Phi_{1}\left[\int_{0}^{t} v(s) d s\right]=0\right\}
$$

If $v \in \operatorname{Im} L$, then there exists $u \in \operatorname{dom} L$ such that $D_{0+}^{\alpha} u(t)=v(t)$. Hence,

$$
u(t)=\frac{1}{\Gamma(\alpha)} \int_{0}^{t}(t-s)^{\alpha-1} v(s) d s+a t^{\alpha-1}+b t^{\alpha-2}
$$

for some $a, b \in \mathbb{R}$. It yields

$$
\Phi_{1}\left[D_{0+}^{\alpha-1} u(t)\right]=\Phi_{1}\left[\int_{0}^{t} v(s) d s\right]+a \Gamma(\alpha) \Phi_{1}[1]=\Phi_{1}\left[\int_{0}^{t} v(s) d s\right]=0 .
$$

Therefore

$$
\operatorname{Im} L \subset\left\{v \in Z: \Phi_{1}\left[\int_{0}^{t} v(s) d s\right]=0\right\}
$$

On the other hand, suppose $v \in Z$ satisfies

$$
\Phi_{1}\left[\int_{0}^{t} v(s) d s\right]=0
$$

Let

$$
u(t)=\frac{1}{\Gamma(\alpha)} \int_{0}^{t}(t-s)^{\alpha-1} v(s) d s-\frac{t^{\alpha-2}}{\Gamma(\alpha-1) \Phi_{2}[1]} \Phi_{2}\left[\int_{0}^{t}(t-s) v(s) d s\right]
$$

Obviously, $D_{0+}^{\alpha} u(t)=v(t)$ and $\left.I_{0+}^{3-\alpha} u(t)\right|_{t=0}=0$. Considering (A2) and the linearity of $\Phi_{i}$ $(i=1,2)$, we have

$$
\Phi_{1}\left[D_{0+}^{\alpha-1} u(t)\right]=\Phi_{1}\left[\int_{0}^{t} v(s) d s\right]=0
$$

and

$$
\Phi_{2}\left[D_{0+}^{\alpha-2} u(t)\right]=\Phi_{2}\left[\int_{0}^{t}(t-s) v(s) d s\right]-\Phi_{2}\left[\frac{1}{\Phi_{2}[1]} \Phi_{2}\left[\int_{0}^{t}(t-s) v(s) d s\right]\right]=0 .
$$

It yields

$$
\left\{v \in Z: \Phi_{1}\left[\int_{0}^{t} v(s) d s\right]=0\right\} \subset \operatorname{Im} L
$$

The proof is complete.

Lemma 2.6 If $\Phi_{1}[t] \neq 0$, then: 
(i) $L$ is a Fredholm operator of index zero and $\operatorname{dim} \operatorname{Ker} L=\operatorname{codim} \operatorname{Im} L=1$.

(ii) The linear operator $K_{p}: \operatorname{Im} L \rightarrow \operatorname{dom} L \cap \operatorname{Ker} P$ can be defined by

$$
\left(K_{p} v\right)(t)=I_{0+}^{\alpha} v(t)-\frac{t^{\alpha-2}}{\Gamma(\alpha-1) \Phi_{2}[1]} \Phi_{2}\left[\int_{0}^{t}(t-s) v(s) d s\right]
$$

(iii) $\left\|K_{p} v\right\|_{C^{\alpha-1}} \leq \Delta_{1}\|v\|_{1}$, where $\Delta_{1}=2+\frac{1}{\Gamma(\alpha)}+\frac{(1+\Gamma(\alpha-1))\left\|\Phi_{2}\right\|}{\Gamma(\alpha-1)\left|\Phi_{2}[1]\right|}$ and $\left\|\Phi_{2}\right\|$ is the norm of $a$ continuous linear functional $\Phi_{2}$.

(iv) The linear operator $K_{p}: \operatorname{Im} L \rightarrow \operatorname{dom} L \cap \operatorname{Ker} P \subset C^{\alpha-1}[0,1]$ is completely continuous.

Proof Firstly, we construct the mapping $Q: Z \rightarrow Z$ defined by

$$
Q y=\frac{1}{\Phi_{1}[t]} \Phi_{1}\left[\int_{0}^{t} y(s) d s\right]
$$

Noting that

$$
Q^{2} y=\frac{1}{\Phi_{1}[t]} \Phi_{1}\left[\int_{0}^{t}(Q y) d s\right]=\frac{1}{\Phi_{1}[t]} \Phi_{1}\left[\int_{0}^{t} d s\right](Q y)=Q y
$$

we get $Q: Z \rightarrow Z$ is a well-defined projector.

Now, it is obvious that $\operatorname{Im} L=\operatorname{Ker} Q$. Noting that $Q$ is a linear projector, we have $Z=$ $\operatorname{Im} Q \oplus \operatorname{Ker} Q$. Hence, $Z=\operatorname{Im} Q \oplus \operatorname{Im} L$ and $\operatorname{dim} \operatorname{Ker} L=\operatorname{codim} \operatorname{Im} L=1$. This means $L$ is a Fredholm mapping of index zero. Taking $P: Y \rightarrow Y$ as

$$
(P u)(t)=\frac{\left.D_{0+}^{\alpha-1} u(t)\right|_{t=0}}{\Gamma(\alpha)} t^{\alpha-1}
$$

then the generalized inverse $K_{p}: \operatorname{Im} L \rightarrow \operatorname{dom} L \cap \operatorname{Ker} P$ of $L$ can be rewritten

$$
\left(K_{p} v\right)(t)=I_{0+}^{\alpha} v(t)-\frac{t^{\alpha-2}}{\Gamma(\alpha-1) \Phi_{2}[1]} \Phi_{2}\left[\int_{0}^{t}(t-s) v(s) d s\right] .
$$

In fact, for $v \in \operatorname{Im} L$, we have

$$
\begin{aligned}
& \left.I_{0+}^{3-\alpha}\left(K_{p} v\right)(t)\right|_{t=0}=0, \\
& \Phi_{1}\left[D_{0+}^{\alpha-1}\left(K_{p} v\right)(t)\right]=\Phi_{1}\left[D_{0+}^{\alpha-1} I_{0+}^{\alpha} v(t)\right]=\Phi_{1}\left[\int_{0}^{t} v(s) d s\right]=0
\end{aligned}
$$

and

$$
\Phi_{2}\left[D_{0+}^{\alpha-2}\left(K_{p} v\right)(t)\right]=\Phi_{2}\left[\int_{0}^{t}(t-s) v(s) d s\right]-\frac{1}{\Phi_{2}[1]} \Phi_{2}\left[\int_{0}^{t}(t-s) v(s) d s\right] \Phi_{2}[1]=0
$$

which implies that $K_{p}$ is well defined on $\operatorname{Im} L$. Moreover, for $v \in \operatorname{Im} L$, we have

$$
\left(L K_{p}\right) v(t)=D_{0+}^{\alpha} I_{0+}^{\alpha} v(t)=v(t)
$$


Zou and Gui Advances in Difference Equations 2013, 2013:233

Page 9 of 25

http://www.advancesindifferenceequations.com/content/2013/1/233

and for $v \in \operatorname{dom} L \cap \operatorname{Ker} P$, we know

$$
\begin{array}{r}
I_{0+}^{\alpha} D_{0+}^{\alpha} v(t)=v(t)-\frac{\left.D_{0+}^{\alpha-1} v(t)\right|_{t=0}}{\Gamma(\alpha)} t^{\alpha-1}-\frac{\left.D_{0+}^{\alpha-2} v(t)\right|_{t=0}}{\Gamma(\alpha-1)} t^{\alpha-2}-\frac{\left.I_{0+}^{3-\alpha} v(t)\right|_{t=0}}{\Gamma(\alpha)} t^{\alpha-3}, \\
v \in \operatorname{dom} L \cap \operatorname{Ker} P \text { means that }\left.I_{0+}^{3-\alpha} v(t)\right|_{t=0}=\left.D_{0+}^{\alpha-1} v(t)\right|_{t=0}=\Phi_{2}\left[D_{0+}^{\alpha-2} v(t)\right]=0 . \text { So, } \\
\left(K_{p} L\right) v(t)=I_{0+}^{\alpha} D_{0+}^{\alpha} v(t)-\frac{t^{\alpha-2}}{\Gamma(\alpha-1) \Phi_{2}[1]} \Phi_{2}\left[D_{0+}^{\alpha-2} v(t)-\left.D_{0+}^{\alpha-2} v(t)\right|_{t=0}\right] \\
=v(t)-\frac{\left.D_{0+}^{\alpha-2} v(t)\right|_{t=0}}{\Gamma(\alpha-1)} t^{\alpha-2}+\frac{t^{\alpha-2}}{\Gamma(\alpha-1) \Phi_{2}[1]} \Phi_{2}\left[\left.D_{0+}^{\alpha-2} v(t)\right|_{t=0}\right]=v(t) .
\end{array}
$$

That is, $K_{p}=\left(\left.L\right|_{\operatorname{dom} L \cap \operatorname{Ker} P}\right)^{-1}$. Since

$$
\begin{aligned}
& D_{0+}^{\alpha-1}\left(K_{p} v\right)(t)=\int_{0}^{t} v(s) d s, \\
& D_{0+}^{\alpha-2}\left(K_{p} v\right)(t)=\int_{0}^{t}(t-s) v(s) d s-\frac{1}{\Phi_{2}[1]} \Phi_{2}\left[\int_{0}^{t}(t-s) v(s) d s\right],
\end{aligned}
$$

then

$$
\begin{aligned}
& \left\|K_{p} v\right\|_{\infty} \leq\left(\frac{1}{\Gamma(\alpha)}+\frac{\left\|\Phi_{2}\right\|}{\Gamma(\alpha-1)\left|\Phi_{2}[1]\right|}\right)\|v\|_{1}, \\
& \left\|D_{0+}^{\alpha-1}\left(K_{p} v\right)\right\|_{\infty} \leq\|v\|_{1}, \quad\left\|D_{0+}^{\alpha-2}\left(K_{p} v\right)\right\|_{\infty} \leq\left(1+\frac{\left\|\Phi_{2}\right\|}{\left|\Phi_{2}[1]\right|}\right)\|v\|_{1} .
\end{aligned}
$$

It follows that

$$
\left\|K_{p} v\right\|_{C^{\alpha-1}} \leq\left(2+\frac{1}{\Gamma(\alpha)}+\frac{(1+\Gamma(\alpha-1))\left\|\Phi_{2}\right\|}{\Gamma(\alpha-1)\left|\Phi_{2}[1]\right|}\right)\|v\|_{1} .
$$

Finally, we prove that $K_{p}: \operatorname{Im} L \rightarrow \operatorname{dom} L \cap \operatorname{Ker} P \subset C^{\alpha-1}[0,1]$ is completely continuous. Let $V \subset \operatorname{Im} L \subset L^{1}[0,1]$ be a bounded set. From the above discussion, we only need to prove that $K_{p} V$ is equicontinuous on [0,1]. For $v \in V, t_{1}, t_{2} \in[0,1]$ with $t_{1}<t_{2}$, we have

$$
\begin{aligned}
\left|D_{0+}^{\alpha-1}\left(K_{p} v\right)\left(t_{1}\right)-D_{0+}^{\alpha-1}\left(K_{p} v\right)\left(t_{2}\right)\right| & =\left|\int_{t_{2}}^{t_{1}} v(s) d s\right| \leq \int_{t_{2}}^{t_{1}}|v(s)| d s, \\
\left|D_{0+}^{\alpha-2}\left(K_{p} v\right)\left(t_{1}\right)-D_{0+}^{\alpha-2}\left(K_{p} v\right)\left(t_{2}\right)\right| & =\left|\int_{0}^{t_{1}}\left(t_{1}-s\right) v(s) d s-\int_{0}^{t_{2}}\left(t_{2}-s\right) v(s) d s\right| \\
& \leq\left|\int_{t_{2}}^{t_{1}}\left(t_{1}-s\right) v(s) d s\right|+\left|\int_{0}^{t_{2}}\left(t_{1}-t_{2}\right) v(s) d s\right| \\
& \leq \int_{t_{2}}^{t_{1}}|v(s)| d s+\left(t_{1}-t_{2}\right)\|v\|_{1}
\end{aligned}
$$

and

$$
\begin{aligned}
& \left|\left(K_{p} v\right)\left(t_{1}\right)-\left(K_{p} v\right)\left(t_{2}\right)\right| \\
& \quad \leq \frac{1}{\Gamma(\alpha)}\left|\int_{0}^{t_{1}}\left(t_{1}-s\right)^{\alpha-1} v(s) d s-\int_{0}^{t_{2}}\left(t_{2}-s\right)^{\alpha-1} v(s) d s\right|
\end{aligned}
$$




$$
\begin{aligned}
& +\frac{\left|\Phi_{2}\left[\int_{0}^{t}(t-s) v(s) d s\right]\right|}{\Gamma(\alpha-1)\left|\Phi_{2}[1]\right|}\left|t_{1}{ }^{\alpha-2}-t_{2}{ }^{\alpha-2}\right| \\
\leq & \frac{1}{\Gamma(\alpha)}\left|\int_{t_{2}}^{t_{1}}\left(t_{1}-s\right)^{\alpha-1} v(s) d s\right|+\frac{1}{\Gamma(\alpha)}\left|\int_{0}^{t_{2}}\left(\left(t_{1}-s\right)^{\alpha-1}-\left(t_{2}-s\right)^{\alpha-1}\right) v(s) d s\right| \\
& +\frac{\left\|\Phi_{2}\right\|\|v\|_{1}}{\Gamma(\alpha-1)\left|\Phi_{2}[1]\right|}\left|t_{1}{ }^{\alpha-2}-t_{2}{ }^{\alpha-2}\right| \\
\leq & \frac{1}{\Gamma(\alpha)} \int_{t_{2}}^{t_{1}}|v(s)| d s+\frac{\alpha-1}{\Gamma(\alpha)}\|v\|_{1}\left(t_{1}-t_{2}\right)+\frac{\left\|\Phi_{2}\right\|\|v\|_{1}}{\Gamma(\alpha-1)\left|\Phi_{2}[1]\right|}\left|t_{1}{ }^{\alpha-2}-t_{2}{ }^{\alpha-2}\right| .
\end{aligned}
$$

Therefore, $K_{p}(V)$ is equicontinuous. Thus, the operator $K_{p}: \operatorname{Im} L \rightarrow \operatorname{dom} L \cap \operatorname{Ker} P$ is completely continuous. The proof is complete.

Similar to Lemmas 2.5 and 2.6, we can obtain the following lemma.

Lemma 2.7 If (A3) holds, then $\operatorname{Ker} L=\left\{a t^{\alpha-2}: a \in \mathbb{R}\right\}$ and

$$
\operatorname{Im} L=\left\{v: \Phi_{1}\left[\int_{0}^{t} v(s) d s\right]=0\right\}
$$

Furthermore, if $\Phi_{1}[t] \neq 0$ also holds, then $L$ is a Fredholm operator of index zero and $\operatorname{dim} \operatorname{Ker} L=\operatorname{codim} \operatorname{Im} L=1$. Here, the projectors $P: Y \rightarrow Y, Q: Z \rightarrow Z$ can be defined as follows:

$$
\begin{aligned}
& (P v)(t)=\frac{\left.D_{0+}^{\alpha-2} v(t)\right|_{t=0}}{\Gamma(\alpha-1)} t^{\alpha-2}, \\
& (Q v)(t)=\frac{\Phi_{1}\left[\int_{0}^{t} v(s) d s\right]}{\Phi_{1}[t]}
\end{aligned}
$$

The generalized inverse operator of $L, K_{P}: \operatorname{Im} L \rightarrow \operatorname{dom} L \cap \operatorname{Ker} P$ can be defined by

$$
\left(K_{p} v\right)(t)=I_{0+}^{\alpha} v(t)-\frac{\Phi_{2}\left[\int_{0}^{t}(t-s) v(s) d s\right]}{\Gamma(\alpha) \Phi_{2}[t]} t^{\alpha-1}
$$

Also,

$$
\left\|K_{p} v\right\|_{C^{\alpha-1}} \leq \Delta_{2}\|v\|_{1}
$$

where $\Delta_{2}=2+\frac{1}{\Gamma(\alpha)}+\frac{(1+2 \Gamma(\alpha))\left\|\Phi_{2}\right\|}{\Gamma(\alpha)\left|\Phi_{2}[t]\right|}$.

Lemma 2.8 If (A4) holds, then $\operatorname{Ker} L=\left\{a t^{\alpha-2}: a \in \mathbb{R}\right\}$ and

$$
\operatorname{Im} L=\left\{v: \Phi_{2}\left[\int_{0}^{t}(t-s) v(s) d s\right]=0\right\}
$$

Furthermore, if $\Phi_{2}\left[t^{2}\right] \neq 0$ also holds, then $L$ is a Fredholm operator of index zero and $\operatorname{dim} \operatorname{Ker} L=\operatorname{codim} \operatorname{Im} L=1$. Here, the projectors $P: Y \rightarrow Y, Q: Z \rightarrow Z$ can be defined as 
follows:

$$
\begin{aligned}
(P v)(t) & =\frac{\left.D_{0+}^{\alpha-2} v\right|_{t=0}}{\Gamma(\alpha-1)} t^{\alpha-2}, \\
(Q v)(t) & =\frac{2 \Phi_{2}\left[\int_{0}^{t}(t-s) v(s) d s\right]}{\Phi_{2}\left[t^{2}\right]} .
\end{aligned}
$$

The generalized inverse operator of $L, K_{P}: \operatorname{Im} L \rightarrow \operatorname{dom} L \cap \operatorname{Ker} P$ can be defined by

$$
\left(K_{p} v\right)(t)=I_{0+}^{\alpha} v(t)-\frac{\Phi_{1}\left[\int_{0}^{t} v(s) d s\right]}{\Gamma(\alpha) \Phi_{1}[1]} t^{\alpha-1}
$$

Also,

$$
\left\|K_{p} v\right\|_{C^{\alpha-1}} \leq \Delta_{3}\|v\|_{1}
$$

where $\Delta_{3}=2+\frac{1}{\Gamma(\alpha)}+\frac{(1+2 \Gamma(\alpha))\left\|\Phi_{1}\right\|}{\Gamma(\alpha)\left|\Phi_{1}[1]\right|}$ and $\left\|\Phi_{1}\right\|$ is the norm of the continuous linear functional $\Phi_{1}$.

Lemma 2.9 If (A5) holds, then

$$
\operatorname{Ker} L=\left\{u \in \operatorname{dom} L: u=a t^{\alpha-1}+b t^{\alpha-2}, a, b \in \mathbb{R}, t \in[0,1]\right\}
$$

and

$$
\operatorname{Im} L=\left\{v \in Z: \Phi_{1}\left[\int_{0}^{t} v(s) d s\right]=\Phi_{2}\left[\int_{0}^{t}(t-s) v(s) d s\right]=0\right\}
$$

Proof Let $u(t)=a t^{\alpha-1}+b t^{\alpha-2}$. Clearly, $D_{0+}^{\alpha} u(t)=0$ and $\left.I_{0+}^{3-\alpha} u(t)\right|_{t=0}=0$. Considering (A5), $\Phi_{1}\left[D_{0+}^{\alpha-1} u(t)\right]=\Phi_{1}[\Gamma(\alpha)]=\Gamma(\alpha) \Phi_{1}[1]=0$ and $\Phi_{2}\left[D_{0+}^{\alpha-2} u(t)\right]=a \Gamma(\alpha) \Phi_{2}[t]+b \Gamma(\alpha-$ 1) $\Phi_{2}[1]=0$. So,

$$
\left\{u \in \operatorname{dom} L: u=a t^{\alpha-1}+b t^{\alpha-2}, a, b \in \mathbb{R}, t \in[0,1]\right\} \subset \operatorname{Ker} L .
$$

If $L u=D_{0+}^{\alpha} u(t)=0$, then $u(t)=a t^{\alpha-1}+b t^{\alpha-2}+c t^{\alpha-3}$. Considering $D_{0+}^{\alpha} u(t)=0$ and (A5), we can obtain that

$$
\operatorname{Ker} L \subset\left\{u \in \operatorname{dom} L: u=a t^{\alpha-1}+b t^{\alpha-2}, a, b \in \mathbb{R}, t \in[0,1]\right\} .
$$

We now show that

$$
\operatorname{Im} L=\left\{v \in Z: \Phi_{1}\left[\int_{0}^{t} v(s) d s\right]=\Phi_{2}\left[\int_{0}^{t}(t-s) v(s) d s\right]=0\right\} .
$$

If $v \in \operatorname{Im} L$, then there exists $u \in \operatorname{dom} L$ such that $D_{0+}^{\alpha} u(t)=v(t)$. Hence,

$$
u(t)=\frac{1}{\Gamma(\alpha)} \int_{0}^{t}(t-s)^{\alpha-1} v(s) d s+a t^{\alpha-1}+b t^{\alpha-2}
$$


for some $a, b \in \mathbb{R}$. It yields

$$
\Phi_{1}\left[D_{0+}^{\alpha-1} u(t)\right]=\Phi_{1}\left[\int_{0}^{t} v(s) d s\right]+a \Gamma(\alpha) \Phi_{1}[1]=\Phi_{1}\left[\int_{0}^{t} v(s) d s\right]=0
$$

and

$$
\begin{aligned}
\Phi_{2}\left[D_{0+}^{\alpha-2} u(t)\right] & =\Phi_{2}\left[\int_{0}^{t}(t-s) v(s) d s\right]+a \Gamma(\alpha) \Phi_{2}[t]+b \Gamma(\alpha-1) \Phi_{2}[1] \\
& =\Phi_{2}\left[\int_{0}^{t}(t-s) v(s) d s\right]=0 .
\end{aligned}
$$

Therefore,

$$
\operatorname{Im} L \subset\left\{v \in Z: \Phi_{1}\left[\int_{0}^{t} v(s) d s\right]=\Phi_{2}\left[\int_{0}^{t}(t-s) v(s) d s\right]=0\right\} .
$$

On the other hand, suppose $v \in Z$ satisfies

$$
\Phi_{1}\left[\int_{0}^{t} v(s) d s\right]=\Phi_{2}\left[\int_{0}^{t}(t-s) v(s) d s\right]=0
$$

Let

$$
u(t)=I_{0+}^{\alpha} v(t)=\frac{1}{\Gamma(\alpha)} \int_{0}^{t}(t-s)^{\alpha-1} v(s) d s
$$

Obviously, $D_{0+}^{\alpha} u(t)=v(t)$ and $\left.I_{0+}^{3-\alpha} u(t)\right|_{t=0}=0$. Considering (A5) and the linearity of $\Phi_{i}$ $(i=1,2)$, we have

$$
\Phi_{1}\left[D_{0+}^{\alpha-1} u(t)\right]=\Phi_{1}\left[\int_{0}^{t} v(s) d s\right]=0
$$

and

$$
\Phi_{2}\left[D_{0+}^{\alpha-2} u(t)\right]=\Phi_{2}\left[\int_{0}^{t}(t-s) v(s) d s\right]=0
$$

It yields

$$
\left\{v \in Z: \Phi_{1}\left[\int_{0}^{t} v(s) d s\right]=\Phi_{2}\left[\int_{0}^{t}(t-s) v(s) d s\right]=0\right\} \subset \operatorname{Im} L .
$$

The proof is complete.

Lemma 2.10 If $2 \Phi_{1}[t] \Phi_{2}\left[t^{3}\right]-3 \Phi_{1}\left[t^{2}\right] \Phi_{2}\left[t^{2}\right] \neq 0$, then $L$ is a Fredholm operator of index zero and $\operatorname{dim} \operatorname{Ker} L=\operatorname{codim} \operatorname{Im} L=2$. Furthermore, the linear operator $K_{p}: \operatorname{Im} L \rightarrow \operatorname{dom} L \cap$ Ker $P$ can be defined by

$$
\left(K_{p} v\right)(t)=I_{0+}^{\alpha} v(t) .
$$


Also,

$$
\left\|K_{p} v\right\|_{C^{\alpha-1}} \leq\left(2+\frac{1}{\Gamma(\alpha)}\right)\|v\|_{1}
$$

Proof Firstly, we construct the mapping $Q: Z \rightarrow Z$ defined by

$$
\begin{aligned}
(Q v)(t)= & \frac{2 \Phi_{2}\left[t^{3}\right] \Phi_{1}\left[\int_{0}^{t} v(s) d s\right]-6 \Phi_{1}\left[t^{2}\right] \Phi_{2}\left[\int_{0}^{t}(t-s) v(s) d s\right]}{2 \Phi_{1}[t] \Phi_{2}\left[t^{3}\right]-3 \Phi_{1}\left[t^{2}\right] \Phi_{2}\left[t^{2}\right]} \\
& -\frac{6 \Phi_{2}\left[t^{2}\right] \Phi_{1}\left[\int_{0}^{t} v(s) d s\right]-12 \Phi_{1}[t] \Phi_{2}\left[\int_{0}^{t}(t-s) v(s) d s\right]}{2 \Phi_{1}[t] \Phi_{2}\left[t^{3}\right]-3 \Phi_{1}\left[t^{2}\right] \Phi_{2}\left[t^{2}\right]} t .
\end{aligned}
$$

Let

$$
T_{1} v=\frac{2 \Phi_{2}\left[t^{3}\right] \Phi_{1}\left[\int_{0}^{t} v(s) d s\right]-6 \Phi_{1}\left[t^{2}\right] \Phi_{2}\left[\int_{0}^{t}(t-s) v(s) d s\right]}{2 \Phi_{1}[t] \Phi_{2}\left[t^{3}\right]-3 \Phi_{1}\left[t^{2}\right] \Phi_{2}\left[t^{2}\right]}
$$

and

$$
T_{2} v=-\frac{6 \Phi_{2}\left[t^{2}\right] \Phi_{1}\left[\int_{0}^{t} v(s) d s\right]-12 \Phi_{1}[t] \Phi_{2}\left[\int_{0}^{t}(t-s) v(s) d s\right]}{2 \Phi_{1}[t] \Phi_{2}\left[t^{3}\right]-3 \Phi_{1}\left[t^{2}\right] \Phi_{2}\left[t^{2}\right]} .
$$

We have

$$
Q v=T_{1} v+\left(T_{2} v\right) t
$$

Noting that

$$
\begin{aligned}
T_{1}\left(T_{1} v\right) & =\frac{2 \Phi_{2}\left[t^{3}\right] \Phi_{1}\left[\int_{0}^{t}\left(T_{1} v\right) d s\right]-6 \Phi_{1}\left[t^{2}\right] \Phi_{2}\left[\int_{0}^{t}(t-s)\left(T_{1} v\right) d s\right]}{2 \Phi_{1}[t] \Phi_{2}\left[t^{3}\right]-3 \Phi_{1}\left[t^{2}\right] \Phi_{2}\left[t^{2}\right]} \\
& =\frac{2 \Phi_{2}\left[t^{3}\right] \Phi_{1}\left[\int_{0}^{t} d s\right]-6 \Phi_{1}\left[t^{2}\right] \Phi_{2}\left[\int_{0}^{t}(t-s) d s\right]}{2 \Phi_{1}[t] \Phi_{2}\left[t^{3}\right]-3 \Phi_{1}\left[t^{2}\right] \Phi_{2}\left[t^{2}\right]}\left(T_{1} v\right) \\
& =\frac{2 \Phi_{2}\left[t^{3}\right] \Phi_{1}[t]-6 \Phi_{1}\left[t^{2}\right] \Phi_{2}\left[\frac{t^{2}}{2}\right]}{2 \Phi_{1}[t] \Phi_{2}\left[t^{3}\right]-3 \Phi_{1}\left[t^{2}\right] \Phi_{2}\left[t^{2}\right]}\left(T_{1} v\right) \\
& =T_{1} v, \\
T_{1}\left(T_{2} v\right) & =\frac{2 \Phi_{2}\left[t^{3}\right] \Phi_{1}\left[\int_{0}^{t}\left(T_{2} v\right) s d s\right]-6 \Phi_{1}\left[t^{2}\right] \Phi_{2}\left[\int_{0}^{t}(t-s)\left(T_{2} v\right) s d s\right]}{2 \Phi_{1}[t] \Phi_{2}\left[t^{3}\right]-3 \Phi_{1}\left[t^{2}\right] \Phi_{2}\left[t^{2}\right]} \\
& =\frac{2 \Phi_{2}\left[t^{3}\right] \Phi_{1}\left[\int_{0}^{t} s d s\right]-6 \Phi_{1}\left[t^{2}\right] \Phi_{2}\left[\int_{0}^{t}(t-s) s d s\right]}{2 \Phi_{1}[t] \Phi_{2}\left[t^{3}\right]-3 \Phi_{1}\left[t^{2}\right] \Phi_{2}\left[t^{2}\right]}\left(T_{2} v\right) \\
& =\frac{2 \Phi_{2}\left[t^{3}\right] \Phi_{1}\left[\frac{t^{2}}{2}\right]-6 \Phi_{1}\left[t^{2}\right] \Phi_{2}\left[\frac{t^{3}}{6}\right]}{2 \Phi_{1}[t] \Phi_{2}\left[t^{3}\right]-3 \Phi_{1}\left[t^{2}\right] \Phi_{2}\left[t^{2}\right]}\left(T_{2} v\right) \\
& =0, \\
T_{2}\left(T_{1} v\right) & =-\frac{6 \Phi_{2}\left[t^{2}\right] \Phi_{1}\left[\int_{0}^{t}\left(T_{1} v\right) d s\right]-12 \Phi_{1}[t] \Phi_{2}\left[\int_{0}^{t}(t-s)\left(T_{1} v\right) d s\right]}{2 \Phi_{1}[t] \Phi_{2}\left[t^{3}\right]-3 \Phi_{1}\left[t^{2}\right] \Phi_{2}\left[t^{2}\right]} \\
& =-\frac{6 \Phi_{2}\left[t^{2}\right] \Phi_{1}\left[\int_{0}^{t} d s\right]-12 \Phi_{1}[t] \Phi_{2}\left[\int_{0}^{t}(t-s) d s\right]}{2 \Phi_{1}[t] \Phi_{2}\left[t^{3}\right]-3 \Phi_{1}\left[t^{2}\right] \Phi_{2}\left[t^{2}\right]}\left(T_{1} v\right)
\end{aligned}
$$




$$
\begin{aligned}
& =-\frac{6 \Phi_{2}\left[t^{2}\right] \Phi_{1}[t]-12 \Phi_{1}[t] \Phi_{2}\left[\frac{t^{2}}{2}\right]}{2 \Phi_{1}[t] \Phi_{2}\left[t^{3}\right]-3 \Phi_{1}\left[t^{2}\right] \Phi_{2}\left[t^{2}\right]}\left(T_{1} v\right) \\
& =0
\end{aligned}
$$

and

$$
\begin{aligned}
T_{2}\left(T_{2} v\right) & =-\frac{6 \Phi_{2}\left[t^{2}\right] \Phi_{1}\left[\int_{0}^{t}\left(T_{2} v\right) s d s\right]-12 \Phi_{1}[t] \Phi_{2}\left[\int_{0}^{t}(t-s)\left(T_{2} v\right) s d s\right]}{2 \Phi_{1}[t] \Phi_{2}\left[t^{3}\right]-3 \Phi_{1}\left[t^{2}\right] \Phi_{2}\left[t^{2}\right]} \\
& =-\frac{6 \Phi_{2}\left[t^{2}\right] \Phi_{1}\left[\int_{0}^{t} s d s\right]-12 \Phi_{1}[t] \Phi_{2}\left[\int_{0}^{t}(t-s) s d s\right]}{2 \Phi_{1}[t] \Phi_{2}\left[t^{3}\right]-3 \Phi_{1}\left[t^{2}\right] \Phi_{2}\left[t^{2}\right]}\left(T_{2} v\right) \\
& =-\frac{6 \Phi_{2}\left[t^{2}\right] \Phi_{1}\left[\frac{t^{2}}{2}\right]-12 \Phi_{1}[t] \Phi_{2}\left[\frac{t^{3}}{6}\right]}{2 \Phi_{1}[t] \Phi_{2}\left[t^{3}\right]-3 \Phi_{1}\left[t^{2}\right] \Phi_{2}\left[t^{2}\right]}\left(T_{2} v\right) \\
& =T_{2} v
\end{aligned}
$$

we have, for each $v \in Z$, that

$$
Q^{2} v=T_{1}\left(T_{1} v+\left(T_{2} v\right) t\right)+T_{2}\left(T_{1} v+\left(T_{2} v\right) t\right) t=T_{1} v+\left(T_{2} v\right) t=Q v
$$

So, $Q: Z \rightarrow Z$ is a well-defined projector.

Now we will show that $\operatorname{Ker} Q=\operatorname{Im} L$. If $v \in \operatorname{Ker} Q$, from $Q v=0$, we have $T_{1} v=0$ and $T_{2} v=0$. Considering the definitions of $T_{1}$ and $T_{2}$, we have

$$
\left\{\begin{array}{l}
\Phi_{2}\left[t^{3}\right] \Phi_{1}\left[\int_{0}^{t} v(s) d s\right]-3 \Phi_{1}\left[t^{2}\right] \Phi_{2}\left[\int_{0}^{t}(t-s) v(s) d s\right]=0 \\
\Phi_{2}\left[t^{2}\right] \Phi_{1}\left[\int_{0}^{t} v(s) d s\right]-2 \Phi_{1}[t] \Phi_{2}\left[\int_{0}^{t}(t-s) v(s) d s\right]=0
\end{array}\right.
$$

Since

$$
\left|\begin{array}{ll}
\Phi_{2}\left[t^{3}\right] & -3 \Phi_{1}\left[t^{2}\right] \\
\Phi_{2}\left[t^{2}\right] & -2 \Phi_{1}[t]
\end{array}\right|=-2 \Phi_{1}[t] \Phi_{2}\left[t^{3}\right]+3 \Phi_{1}\left[t^{2}\right] \Phi_{2}\left[t^{2}\right] \neq 0
$$

so $\Phi_{1}\left[\int_{0}^{t} v(s) d s\right]=\Phi_{2}\left[\int_{0}^{t}(t-s) v(s) d s\right]=0$, which yields $v \in \operatorname{Im} L$. On the other hand, if $v \in \operatorname{Im} L$, from $\Phi_{1}\left[\int_{0}^{t} v(s) d s\right]=\Phi_{2}\left[\int_{0}^{t}(t-s) v(s) d s\right]=0$ and the definition of $Q$, it is obvious that $Q v=0$, thus $v \in \operatorname{Ker} Q$. Hence, $\operatorname{Ker} Q=\operatorname{Im} L$.

For $v \in Z$, from $v=(v-Q v)+Q v, v-Q v \in \operatorname{Ker} Q=\operatorname{Im} L, Q v \in \operatorname{Im} Q$, we have $Z=\operatorname{Im} L+$ $\operatorname{Im} Q$. And for any $v \in \operatorname{Im} L \cap \operatorname{Im} Q$, from $v \in \operatorname{Im} Q$, there exist constants $a, b \in \mathbb{R}$ such that $v(t)=a+b t$. From $v \in \operatorname{Im} L$, we obtain

$$
\left\{\begin{array}{l}
\Phi_{1}[t] \cdot a+\Phi_{1}\left[\frac{t^{2}}{2}\right] \cdot b=0 \\
\Phi_{2}\left[\frac{t^{2}}{2}\right] \cdot a+\Phi_{2}\left[\frac{t^{3}}{6}\right] \cdot b=0
\end{array}\right.
$$

In view of

$$
\left|\begin{array}{cc}
\Phi_{1}[t] & \Phi_{1}\left[\frac{t^{2}}{2}\right] \\
\Phi_{2}\left[\frac{t^{2}}{2}\right] & \Phi_{2}\left[\frac{t^{3}}{6}\right]
\end{array}\right|=\frac{1}{6} \Phi_{1}[t] \Phi_{2}\left[t^{3}\right]-\frac{1}{4} \Phi_{1}\left[t^{2}\right] \Phi_{2}\left[t^{2}\right] \neq 0,
$$


therefore (2.8) has a unique solution $a=b=0$, which implies $\operatorname{Im} L \cap \operatorname{Im} Q=\{\theta\}$ and $Z=$ $\operatorname{Im} L \oplus \operatorname{Im} Q$. Since $\operatorname{dim} \operatorname{Ker} L=\operatorname{dim} \operatorname{Im} Q=\operatorname{codim} \operatorname{Im} L=2$, thus $L$ is a Fredholm map of index zero. Let $P: Y \rightarrow Y$ be defined by

$$
(P v)(t)=\frac{\left.D_{0+}^{\alpha-1} u(t)\right|_{t=0}}{\Gamma(\alpha)} t^{\alpha-1}+\frac{\left.D_{0+}^{\alpha-2} u(t)\right|_{t=0}}{\Gamma(\alpha-1)} t^{\alpha-2} .
$$

Then the generalized inverse $K_{p}: \operatorname{Im} L \rightarrow \operatorname{dom} L \cap \operatorname{Ker} P$ of $L$ can be rewritten

$$
\left(K_{p} v\right)(t)=I_{0+}^{\alpha} v(t) .
$$

In fact, for $v \in \operatorname{Im} L$, we have

$$
\begin{aligned}
& \left.I_{0+}^{3-\alpha}\left(K_{p} v\right)(t)\right|_{t=0}=0, \\
& \Phi_{1}\left[D_{0+}^{\alpha-1}\left(K_{p} v\right)(t)\right]=\Phi_{1}\left[D_{0+}^{\alpha-1} I_{0+}^{\alpha} v(t)\right]=\Phi_{1}\left[\int_{0}^{t} v(s) d s\right]=0
\end{aligned}
$$

and

$$
\Phi_{2}\left[D_{0+}^{\alpha-2}\left(K_{p} v\right)(t)\right]=\Phi_{2}\left[\int_{0}^{t}(t-s) v(s) d s\right]=0,
$$

which implies that $K_{p}$ is well defined on $\operatorname{Im} L$. Moreover, for $v \in \operatorname{Im} L$, we have

$$
\left(L K_{p}\right) v(t)=D_{0+}^{\alpha} I_{0+}^{\alpha} \nu(t)=v(t)
$$

and for $v \in \operatorname{dom} L \cap \operatorname{Ker} P$, we know

$$
I_{0+}^{\alpha} D_{0+}^{\alpha} \nu(t)=v(t)-\frac{\left.D_{0+}^{\alpha-1} v(t)\right|_{t=0}}{\Gamma(\alpha)} t^{\alpha-1}-\frac{\left.D_{0+}^{\alpha-2} v(t)\right|_{t=0}}{\Gamma(\alpha-1)} t^{\alpha-2}-\frac{\left.I_{0+}^{3-\alpha} v(t)\right|_{t=0}}{\Gamma(\alpha)} t^{\alpha-3},
$$

$v \in \operatorname{dom} L \cap \operatorname{Ker} P$ means that $\left.I_{0+}^{3-\alpha} v(t)\right|_{t=0}=\left.D_{0+}^{\alpha-1} v(t)\right|_{t=0}=\left.D_{0+}^{\alpha-2} v(t)\right|_{t=0}=0$. So,

$$
\left(K_{p} L\right) v(t)=I_{0+}^{\alpha} D_{0+}^{\alpha} v(t)=v(t) .
$$

That is, $K_{p}=\left(\left.L\right|_{\operatorname{dom} L \cap \operatorname{Ker} P}\right)^{-1}$. Since

$$
\begin{aligned}
& D_{0+}^{\alpha-1}\left(K_{p} v\right)(t)=\int_{0}^{t} v(s) d s, \\
& D_{0+}^{\alpha-2}\left(K_{p} v\right)(t)=\int_{0}^{t}(t-s) v(s) d s,
\end{aligned}
$$

then

$$
\begin{aligned}
& \left\|K_{p} v\right\|_{\infty} \leq \frac{1}{\Gamma(\alpha)}\|v\|_{1}, \\
& \left\|D_{0_{+}}^{\alpha-1}\left(K_{p} v\right)\right\|_{\infty} \leq\|v\|_{1}, \quad\left\|D_{0_{+}}^{\alpha-2}\left(K_{p} v\right)\right\|_{\infty} \leq\|v\|_{1} .
\end{aligned}
$$


It follows that

$$
\left\|K_{p} v\right\|_{C^{\alpha-1}} \leq\left(2+\frac{1}{\Gamma(\alpha)}\right)\|v\|_{1}
$$

The proof is complete.

\section{Main results}

From Lemma 2.4, we can obtain the existence theorem for FBVP (1.1), (1.2).

Theorem 3.1 Assume that (A1) and the following conditions hold:

$$
\left|f\left(t, x_{1}, x_{2}, x_{3}\right)-f\left(t, y_{1}, y_{2}, y_{3}\right)\right| \leq \beta\left(\left|x_{1}-y_{1}\right|+\left|x_{2}-y_{2}\right|+\left|x_{3}-y_{3}\right|\right) \text {. }
$$

Then FBVP (1.1), (1.2) has a unique solution in $C^{\alpha-1}[0,1]$ provided that

$$
\beta\left(2+\frac{\left\|\Phi_{2}\right\|}{\left|\Phi_{2}[1]\right|}+\frac{1}{\Gamma(\alpha)}+\frac{\left\|\Phi_{2}\right\|}{\Gamma(\alpha-1)\left|\Phi_{2}[1]\right|}\right)\left(1+\frac{\left\|\Phi_{1}\right\|}{\left|\Phi_{1}[1]\right|}\right)<1 .
$$

Proof We shall prove that $T x=x$ has a unique solution in $C^{\alpha-1}[0,1]$. For each $u, v \in$ $C^{\alpha-1}[0,1]$, considering the linearity of $\Phi_{i}(i=1,2)$, we have

$$
\begin{aligned}
(T u)(t)-(T v)(t)= & \frac{1}{\Gamma(\alpha)} \int_{0}^{t}(t-s)^{\alpha-1}((\mathbf{f} u)(s)-(\mathbf{f} u)(s)) d s \\
& -\frac{\Phi_{1}\left[\int_{0}^{t}((\mathbf{f} u)(s)-(\mathbf{f} u)(s)) d s\right]}{\Gamma(\alpha) \Phi_{1}[1]} t^{\alpha-1} \\
& -\frac{\Phi_{2}\left[\int_{0}^{t}(t-s)((\mathbf{f} u)(s)-(\mathbf{f} u)(s)) d s\right]}{\Gamma(\alpha-1) \Phi_{2}[1]} t^{\alpha-2} \\
& +\frac{\Phi_{1}\left[\int_{0}^{t}((\mathbf{f} u)(s)-(\mathbf{f} u)(s)) d s\right] \Phi_{2}[t]}{\Gamma(\alpha-1) \Phi_{1}[1] \Phi_{2}[1]} t^{\alpha-2} .
\end{aligned}
$$

Then

$$
\begin{aligned}
& |(T u)(t)-(T v)(t)| \leq \beta\|u-v\|_{C^{\alpha-1}}\left(\frac{1}{\Gamma(\alpha)}+\frac{\left\|\Phi_{2}\right\|}{\Gamma(\alpha-1)\left|\Phi_{2}[1]\right|}\right)\left(1+\frac{\left\|\Phi_{1}\right\|}{\left|\Phi_{1}[1]\right|}\right), \\
& \left|D_{0+}^{\alpha-1}(T u)(t)-D_{0+}^{\alpha-1}(T v)(t)\right| \leq \beta\|u-v\|_{C^{\alpha-1}}\left(1+\frac{\left\|\Phi_{1}\right\|}{\left|\Phi_{1}[1]\right|}\right)
\end{aligned}
$$

and

$$
\left|D_{0+}^{\alpha-2}(T u)(t)-D_{0+}^{\alpha-2}(T v)(t)\right| \leq \beta\|u-v\|_{C^{\alpha-1}}\left(1+\frac{\left\|\Phi_{2}\right\|}{\left|\Phi_{2}[1]\right|}\right)\left(1+\frac{\left\|\Phi_{1}\right\|}{\left|\Phi_{1}[1]\right|}\right) .
$$

So,

$$
\|T u-T v\|_{C^{\alpha-1}} \leq \beta\|u-v\|_{C^{\alpha-1}}\left(2+\frac{\left\|\Phi_{2}\right\|}{\left|\Phi_{2}[1]\right|}+\frac{1}{\Gamma(\alpha)}+\frac{\left\|\Phi_{2}\right\|}{\Gamma(\alpha-1)\left|\Phi_{2}[1]\right|}\right)\left(1+\frac{\left\|\Phi_{1}\right\|}{\left|\Phi_{1}[1]\right|}\right) .
$$

The above inequality implies that $T$ is a contraction. By using Banach's contraction principle, $T x=x$ has a unique solution in $C^{\alpha-1}[0,1]$. From Lemma 2.4, FBVP (1.1), (1.2) has a unique solution in $C^{\alpha-1}[0,1]$. The proof is complete. 
From Lemmas 2.5-2.8 and Theorem 2.1, we can obtain the existence theorem for FBVP (1.1), (1.2) in the case of $\operatorname{dim} \operatorname{Ker} L=1$.

Theorem 3.2 Let $f:[0,1] \times \mathbb{R}^{3} \rightarrow \mathbb{R}$ be a continuous function. Assume that $\Phi_{1}[t] \neq 0$, (A2) and the following conditions (H1)-(H3) hold:

(H1) There exist functions $\alpha, \beta, \gamma, \omega \in L^{1}[0,1]$ such that for all $(x, y, z) \in \mathbb{R}^{3}, t \in[0,1]$,

$$
|f(t, x, y)| \leq \omega(t)+\alpha(t)|x|+\beta(t)|y|+\gamma(t)|z|
$$

(H2) There exists a constant $A>0$ such that for $u \in \operatorname{dom} L$, if $\left|D_{0+}^{\alpha-1} u(t)\right|>A$ for all $t \in[0,1]$, then $\Phi_{1}\left[\int_{0}^{t} f\left(s, u(s), D_{0+}^{\alpha-1} u(s), D_{0+}^{\alpha-2} u(s)\right) d s\right] \neq 0$.

(H3) There exists a constant $B>0$ such that either for each $a \in \mathbb{R}:|a|>B$,

$$
a \Phi_{1}\left[\int_{0}^{t} f\left(s, a s^{\alpha-1}, a \Gamma(\alpha), 0\right) d s\right]>0
$$

or for each $a \in \mathbb{R}:|a|>B$,

$$
a \Phi_{1}\left[\int_{0}^{t} f\left(s, a s^{\alpha-1}, a \Gamma(\alpha), 0\right) d s\right]<0
$$

Then FBVP (1.1), (1.2) has at least one solution in $C^{\alpha-1}[0,1]$ provided

$$
\left(\frac{1}{\Gamma(\alpha)}+2+\Delta_{1}\right)\left(\|\alpha\|_{1}+\|\beta\|_{1}+\|\gamma\|_{1}\right)<1,
$$

where $\Delta_{1}$ is the same as in Lemma 2.6.

Proof Set

$$
\Omega_{1}=\{u \in \operatorname{dom} L \backslash \operatorname{Ker} L: L u=\lambda N u \text { for some } \lambda \in[0,1]\} .
$$

Then, for $u \in \Omega_{1}$, since $L u=\lambda N u$, so $\lambda \neq 0, N u \in \operatorname{Im} L=\operatorname{Ker} Q$, hence

$$
\Phi_{1}\left[\int_{0}^{t} f\left(s, u(s), D_{0+}^{\alpha-1} u(s), D_{0+}^{\alpha-2} u(s)\right) d s\right]=0 .
$$

Thus, from (H2), there exists $t_{0} \in[0,1]$ such that

$$
\left|D_{0+}^{\alpha-1} u\left(t_{0}\right)\right| \leq A
$$

Now,

$$
D_{0+}^{\alpha-1} u(t)=D_{0+}^{\alpha-1} u\left(t_{0}\right)+\int_{t_{0}}^{t} D_{0+}^{\alpha} u(s) d s,
$$

and so

$$
\begin{aligned}
\left|D_{0+}^{\alpha-1} u(0)\right| & \leq\left\|D_{0+}^{\alpha-1} u(t)\right\|_{\infty} \leq\left|D_{0+}^{\alpha-2} u\left(t_{0}\right)\right|+\left\|D_{0+}^{\alpha} u(t)\right\|_{1} \\
& \leq A+\|L u\|_{1} \leq A+\|N u\|_{1} .
\end{aligned}
$$


Again, for $u \in \Omega_{1}, u \in \operatorname{dom} L \backslash \operatorname{Ker} L$, then $(I-P) u \in \operatorname{dom} L \cap \operatorname{Ker} P$ and $L P u=0$. Thus, from Lemma 2.6, we have

$$
\begin{aligned}
\|(I-P) u\|_{C^{\alpha-1}} & =\left\|K_{P} L(I-P) u\right\|_{C^{\alpha-1}} \leq \Delta_{1}\|L(I-P) u\|_{1} \\
& \leq \Delta_{1}\|N u\|_{1} .
\end{aligned}
$$

From (3.1), (3.2), we have

$$
\begin{aligned}
\|u\|_{C^{\alpha-1}} & \leq\|P u\|_{C^{\alpha-1}}+\|(I-P) u\|_{C^{\alpha-1}} \\
& =\left(\frac{1}{\Gamma(\alpha)}+2\right)\left|D_{0+}^{\alpha-1} u(0)\right|+\|(I-P) u\|_{C^{\alpha-1}} \\
& \leq A\left(\frac{1}{\Gamma(\alpha)}+2\right)+\left(\frac{1}{\Gamma(\alpha)}+2+\Delta_{1}\right)\|N u\|_{1} .
\end{aligned}
$$

By this and (H1), we have

$$
\begin{aligned}
\|u\|_{C^{\alpha-1}} \leq & A\left(\frac{1}{\Gamma(\alpha)}+2\right)+\left(\frac{1}{\Gamma(\alpha)}+2+\Delta_{1}\right)\|\omega\|_{1} \\
& +\left(\frac{1}{\Gamma(\alpha)}+2+\Delta_{1}\right)\left(\|\alpha\|_{1}+\|\beta\|_{1}+\|\gamma\|_{1}\right)\|u\|_{C^{\alpha-1}}
\end{aligned}
$$

and

$$
\|u\|_{C^{\alpha-1}} \leq \frac{A\left(\frac{1}{\Gamma(\alpha)}+2\right)+\left(\frac{1}{\Gamma(\alpha)}+2+\Delta_{1}\right)\|\omega\|_{1}}{1-\left(\frac{1}{\Gamma(\alpha)}+2+\Delta_{1}\right)\left(\|\alpha\|_{1}+\|\beta\|_{1}+\|\gamma\|_{1}\right)} .
$$

Therefore, $\Omega_{1}$ is bounded. Let

$$
\Omega_{2}=\{u \in \operatorname{Ker} L: N u \in \operatorname{Im} L\}
$$

For $u \in \Omega_{2}$, there is $u \in \operatorname{Ker} L=\left\{u \in \operatorname{dom} L \mid u=a t^{\alpha-1}, t \in[0,1], a \in \mathbb{R}\right\}$, and $N u \in \operatorname{Im} L$, thus

$$
\Phi_{1}\left[\int_{0}^{t} f\left(s, a t^{\alpha-1}, a \Gamma(\alpha), a \Gamma(\alpha) s\right) d s\right]=0 .
$$

From (H2), we get $|a| \leq \frac{A}{\Gamma(\alpha)}$, thus $\Omega_{2}$ is bounded.

Next, according to the condition (H3), for any $a \in \mathbb{R}$, if $|a|>B$, then either

$$
a \Phi_{1}\left[\int_{0}^{t} f\left(s, a s^{\alpha-1}, a \Gamma(\alpha), 0\right) d s\right]<0
$$

or else

$$
a \Phi_{1}\left[\int_{0}^{t} f\left(s, a s^{\alpha-1}, a \Gamma(\alpha), 0\right) d s\right]>0 .
$$

If (3.3) holds, set

$$
\Omega_{3}=\{u \in \operatorname{Ker} L:-\lambda J u+(1-\lambda) Q N u=0, \lambda \in[0,1]\},
$$


here $Q$ is given by (2.6) and $J: \operatorname{Ker} L \rightarrow \operatorname{Im} Q$ is the linear isomorphism given by $J\left(a t^{\alpha-1}\right)=$ $\frac{a}{\Phi_{1}[t]}, \forall a \in \mathbb{R}, t \in[0,1]$. For $u=a t^{\alpha-1} \in \Omega_{3}$,

$$
\lambda a=(1-\lambda) \Phi_{1}\left[\int_{0}^{t} f\left(s, a s^{\alpha-1}, a \Gamma(\alpha), 0\right) d s\right] .
$$

If $\lambda=1$, then $a=0$. Otherwise, if $|a|>B$, in view of (3.3), one has

$$
a(1-\lambda) \Phi_{1}\left[\int_{0}^{t} f\left(s, a s^{\alpha-1}, a \Gamma(\alpha), 0\right) d s\right]<0,
$$

which contradicts $\lambda a^{2} \geq 0$. Thus, $\Omega_{3} \subset\left\{u \in \operatorname{Ker} L\left|u=a t^{\alpha-1},\right| a \mid \leq B\right\}$ is bounded.

If (3.4) holds, then define the set

$$
\Omega_{3}=\{x \in \operatorname{Ker} L: \lambda J u+(1-\lambda) Q N u=0, \lambda \in[0,1]\},
$$

here $J$ is as above. Similar to the above argument, we can show that $\Omega_{3}$ is bounded too.

In the following, we shall prove that all the conditions of Theorem 2.1 are satisfied. Let $\Omega$ be a bounded open subset of $Y$ such that $\bigcup_{i=1}^{3} \overline{\Omega_{i}} \subset \Omega$. By Lemma 2.6 and standard arguments, we can prove that $K_{P}(I-Q) N: \Omega \rightarrow Y$ is compact, thus $N$ is $L$-compact on $\bar{\Omega}$. Then, by the above argument, we have

(i) $L u \neq \lambda N u$, for every $(u, \lambda) \in[(\operatorname{dom} L \backslash \operatorname{Ker} L) \cap \partial \Omega] \times(0,1)$,

(ii) $N u \notin \operatorname{Im} L$ for $u \in \operatorname{Ker} L \cap \partial \Omega$.

Finally, we will prove that (iii) of Theorem 2.1 is satisfied. Let $H(u, \lambda)= \pm \lambda J u+(1-\lambda) Q N u$. According to the above argument, we know

$$
H(u, \lambda) \neq 0 \quad \text { for } u \in \operatorname{Ker} L \cap \partial \Omega \text {. }
$$

Thus, by the homotopy property of degree, we have

$$
\begin{aligned}
\operatorname{deg}\left(\left.Q N\right|_{\operatorname{Ker} L}, \operatorname{Ker} L \cap \Omega, 0\right) & =\operatorname{deg}(H(\cdot, 0), \operatorname{Ker} L \cap \Omega, 0) \\
& =\operatorname{deg}(H(\cdot, 1), \operatorname{Ker} L \cap \Omega, 0)=\operatorname{deg}(J, \operatorname{Ker} L \cap \Omega, 0) \neq 0 .
\end{aligned}
$$

Then, by Theorem 2.1, $L u=N u$ has at least one solution in $\operatorname{dom} L \cap \bar{\Omega}$, so that FBVP (1.1), (1.2) has a solution in $C^{\alpha-1}[0,1]$. The proof is complete.

Theorem 3.3 Let $f:[0,1] \times \mathbb{R}^{3} \rightarrow \mathbb{R}$ be a continuous function. Assume that $\Phi_{1}[t] \neq 0$, (A3), (H1) and the following conditions (H4), (H5) hold:

(H4) There exists a constant $A>0$ such that for $u \in \operatorname{dom} L$, if $\left|D_{0+}^{\alpha-1} u(t)\right|+\left|D_{0+}^{\alpha-2} u(t)\right|>A$ for all $t \in[0,1]$, then $\Phi_{1}\left[\int_{0}^{t} f\left(s, u(s), D_{0+}^{\alpha-1} u(s), D_{0+}^{\alpha-2} u(s)\right) d s\right] \neq 0$.

(H5) There exists a constant $B>0$ such that either for each $a \in \mathbb{R}:|a|>B$,

$$
a \Phi_{1}\left[\int_{0}^{t} f\left(s, a s^{\alpha-2}, 0, a \Gamma(\alpha-1)\right) d s\right]>0
$$

or for each $a \in \mathbb{R}:|a|>B$,

$$
a \Phi_{1}\left[\int_{0}^{t} f\left(s, a s^{\alpha-2}, 0, a \Gamma(\alpha-1)\right) d s\right]<0 .
$$


Then FBVP (1.1), (1.2) has at least one solution in $C^{\alpha-1}[0,1]$ provided

$$
\left(\frac{1}{\Gamma(\alpha-1)}+1+\Delta_{2}\right)\left(\|\alpha\|_{1}+\|\beta\|_{1}+\|\gamma\|_{1}\right)<1,
$$

where $\Delta_{2}$ is the same as in Lemma 2.7.

Theorem 3.4 Let $f:[0,1] \times \mathbb{R}^{3} \rightarrow \mathbb{R}$ be a continuous function. Assume that $\Phi_{2}\left[t^{2}\right] \neq 0$, (A4), (H1) and the following conditions (H6), (H7) hold:

(H6) There exists a constant $A>0$ such that for $u \in \operatorname{dom} L$, if $\left|D_{0+}^{\alpha-1} u(t)\right|+\left|D_{0+}^{\alpha-2} u(t)\right|>A$ for all $t \in[0,1]$, then $\Phi_{2}\left[\int_{0}^{t}(t-s) f\left(s, u(s), D_{0+}^{\alpha-1} u(s), D_{0+}^{\alpha-2} u(s)\right) d s\right] \neq 0$.

(H7) There exists a constant $B>0$ such that either for each $a \in \mathbb{R}:|a|>B$,

$$
a \Phi_{2}\left[\int_{0}^{t}(t-s) f\left(s, a s^{\alpha-2}, 0, a \Gamma(\alpha-1)\right) d s\right]>0
$$

or for each $a \in \mathbb{R}:|a|>B$,

$$
a \Phi_{2}\left[\int_{0}^{t}(t-s) f\left(s, a s^{\alpha-2}, 0, a \Gamma(\alpha-1)\right) d s\right]<0
$$

Then FBVP (1.1), (1.2) has at least one solution in $C^{\alpha-1}[0,1]$ provided

$$
\left(\frac{1}{\Gamma(\alpha-1)}+1+\Delta_{3}\right)\left(\|\alpha\|_{1}+\|\beta\|_{1}+\|\gamma\|_{1}\right)<1,
$$

where $\Delta_{3}$ is the same as in Lemma 2.8 .

The proofs of Theorem 3.3 and Theorem 3.4 are similar to that of Theorem 3.2. So, we omit them.

The above Theorem 3.2, Theorem 3.3 and Theorem 3.4 are the existence of solutions to FBVP (1.1), (1.2) in the case of $\operatorname{dim} \operatorname{Ker} L=1$. By making use of Theorem 2.1, Lemma 2.9 and Lemma 2.10, we obtain the existence of solutions for FBVP (1.1), (1.2) in the case of $\operatorname{dim} \operatorname{Ker} L=2$.

Theorem 3.5 Let $f:[0,1] \times \mathbb{R}^{3} \rightarrow \mathbb{R}$ be a continuous function. Assume that $2 \Phi_{1}[t] \Phi_{2}\left[t^{3}\right]-$ $3 \Phi_{1}\left[t^{2}\right] \Phi_{2}\left[t^{2}\right] \neq 0$, (A5), (H1) and the following conditions (H8), (H9) hold:

(H8) There exists a constant $A>0$ such that for $u \in \operatorname{dom} L$, if $\left|D_{0+}^{\alpha-1} u(t)\right|+\left|D_{0+}^{\alpha-2} u(t)\right|>A$ for all $t \in[0,1]$, then

$$
\begin{aligned}
& \Phi_{1}\left[\int_{0}^{t} f\left(s, u(s), D_{0+}^{\alpha-1} u(s), D_{0+}^{\alpha-2} u(s)\right) d s\right] \neq 0 \text { or } \\
& \Phi_{2}\left[\int_{0}^{t}(t-s) f\left(s, u(s), D_{0+}^{\alpha-1} u(s), D_{0+}^{\alpha-2} u(s)\right) d s\right] \neq 0 .
\end{aligned}
$$

(H9) There exists a constant $B>0$ such that for $a_{1}, a_{2} \in \mathbb{R}$ satisfying $\left|a_{1}\right|+\left|a_{2}\right|>B$, either

$$
\begin{aligned}
& a_{1} \Phi_{1}\left[\int_{0}^{t} N\left(a_{1} t^{\alpha-1}+a_{2} t^{\alpha-2}\right) d s\right]>0 \\
& a_{2} \Phi_{2}\left[\int_{0}^{t}(t-s) N\left(a_{1} t^{\alpha-1}+a_{2} t^{\alpha-2}\right) d s\right]>0
\end{aligned}
$$


or

$$
\begin{aligned}
& a_{1} \Phi_{1}\left[\int_{0}^{t} N\left(a_{1} t^{\alpha-1}+a_{2} t^{\alpha-2}\right) d s\right]<0, \\
& a_{2} \Phi_{2}\left[\int_{0}^{t}(t-s) N\left(a_{1} t^{\alpha-1}+a_{2} t^{\alpha-2}\right) d s\right]<0 .
\end{aligned}
$$

Then FBVP (1.1), (1.2) has at least one solution in $C^{\alpha-1}[0,1]$ provided

$$
\left(\frac{2}{\Gamma(\alpha)}+5+\frac{1}{\Gamma(\alpha-1)}\right)\left(\|\alpha\|_{1}+\|\beta\|_{1}+\|\gamma\|_{1}\right)<1
$$

Proof Set

$$
\Omega_{1}=\{u \in \operatorname{dom} L \backslash \operatorname{Ker} L: L u=\lambda N u \text { for some } \lambda \in[0,1]\} .
$$

Then, for $u \in \Omega_{1}$, since $L u=\lambda N u$, so $\lambda \neq 0, N u \in \operatorname{Im} L=\operatorname{Ker} Q$, hence

$$
\Phi_{1}\left[\int_{0}^{t} f\left(s, u(s), D_{0+}^{\alpha-1} u(s), D_{0+}^{\alpha-2} u(s)\right) d s\right]=0
$$

and

$$
\Phi_{2}\left[\int_{0}^{t}(t-s) f\left(s, u(s), D_{0+}^{\alpha-1} u(s), D_{0+}^{\alpha-2} u(s)\right) d s\right]=0 .
$$

Thus, from (H8), there exists $t_{0} \in[0,1]$ such that

$$
\left|D_{0+}^{\alpha-1} u\left(t_{0}\right)\right|+\left|D_{0+}^{\alpha-2} u\left(t_{0}\right)\right| \leq A .
$$

Now,

$$
\begin{aligned}
& D_{0+}^{\alpha-1} u(t)=D_{0+}^{\alpha-1} u\left(t_{0}\right)+\int_{t_{0}}^{t} D_{0+}^{\alpha} u(s) d s, \\
& D_{0+}^{\alpha-2} u(t)=D_{0+}^{\alpha-2} u\left(t_{0}\right)+\int_{t_{0}}^{t} D_{0+}^{\alpha-1} u(s) d s,
\end{aligned}
$$

and so

$$
\begin{aligned}
\left|D_{0+}^{\alpha-1} u(0)\right| & \leq\left\|D_{0+}^{\alpha-1} u(t)\right\|_{\infty} \leq\left|D_{0+}^{\alpha-1} u\left(t_{0}\right)\right|+\left\|D_{0+}^{\alpha} u(t)\right\|_{1} \\
& \leq A+\|L u\|_{1} \leq A+\|N u\|_{1}, \\
\left|D_{0+}^{\alpha-2} u(0)\right| & \leq\left\|D_{0+}^{\alpha-2} u(t)\right\|_{\infty} \leq\left|D_{0+}^{\alpha-2} u\left(t_{0}\right)\right|+\left\|D_{0+}^{\alpha-1} u(t)\right\|_{1} \\
& \leq\left|D_{0+}^{\alpha-2} u\left(t_{0}\right)\right|+\left\|D_{0+}^{\alpha-1} u(t)\right\|_{\infty} \\
& \leq\left|D_{0+}^{\alpha-2} u\left(t_{0}\right)\right|+\left|D_{0+}^{\alpha-1} u\left(t_{0}\right)\right|+\left\|D_{0+}^{\alpha} u(t)\right\|_{1} \leq A+\|N u\|_{1} .
\end{aligned}
$$


Again, for $u \in \Omega_{1}, u \in \operatorname{dom} L \backslash \operatorname{Ker} L$, then $(I-P) u \in \operatorname{dom} L \cap \operatorname{Ker} P$ and $L P u=0$. Thus, from Lemma 2.10, we have

$$
\begin{aligned}
\|(I-P) u\|_{C^{\alpha-1}} & =\left\|K_{P} L(I-P) u\right\|_{C^{\alpha-1}} \leq\left(2+\frac{1}{\Gamma(\alpha)}\right)\|L(I-P) u\|_{1} \\
& \leq\left(2+\frac{1}{\Gamma(\alpha)}\right)\|N u\|_{1} .
\end{aligned}
$$

From (3.7), (3.8) and (3.9), we have

$$
\begin{aligned}
\|u\|_{C^{\alpha-1}} & \leq\|P u\|_{C^{\alpha-1}}+\|(I-P) u\|_{C^{\alpha-1}} \\
& =\left(\frac{1}{\Gamma(\alpha)}+2\right)\left|D_{0+}^{\alpha-1} u(0)\right|+\left(\frac{1}{\Gamma(\alpha-1)}+1\right)\left|D_{0+}^{\alpha-2} u(0)\right|+\|(I-P) u\|_{C^{\alpha-1}} \\
& \leq A\left(\frac{1}{\Gamma(\alpha)}+\frac{1}{\Gamma(\alpha-1)}+3\right)+\left(\frac{2}{\Gamma(\alpha)}+5+\frac{1}{\Gamma(\alpha-1)}\right)\|N u\|_{1} .
\end{aligned}
$$

By this and (H1), we have

$$
\begin{aligned}
\|u\|_{C^{\alpha-1}} \leq & A\left(\frac{1}{\Gamma(\alpha)}+\frac{1}{\Gamma(\alpha-1)}+3\right)+\left(\frac{2}{\Gamma(\alpha)}+5+\frac{1}{\Gamma(\alpha-1)}\right)\|\omega\|_{1} \\
& +\left(\frac{2}{\Gamma(\alpha)}+5+\frac{1}{\Gamma(\alpha-1)}\right)\left(\|\alpha\|_{1}+\|\beta\|_{1}+\|\gamma\|_{1}\right)\|u\|_{C^{\alpha-1}}
\end{aligned}
$$

and

$$
\|u\|_{C^{\alpha-1}} \leq \frac{A\left(\frac{1}{\Gamma(\alpha)}+\frac{1}{\Gamma(\alpha-1)}+3\right)+\left(\frac{2}{\Gamma(\alpha)}+5+\frac{1}{\Gamma(\alpha-1)}\right)\|\omega\|_{1}}{1-\left(\frac{2}{\Gamma(\alpha)}+5+\frac{1}{\Gamma(\alpha-1)}\right)\left(\|\alpha\|_{1}+\|\beta\|_{1}+\|\gamma\|_{1}\right)} .
$$

Therefore, $\Omega_{1}$ is bounded. Let

$$
\Omega_{2}=\{u \in \operatorname{Ker} L: N u \in \operatorname{Im} L\}
$$

For $u \in \Omega_{2}$, there is $u \in \operatorname{Ker} L=\left\{u \in \operatorname{dom} L \mid u=a t^{\alpha-1}+b t^{\alpha-2}, t \in[0,1], a, b \in \mathbb{R}\right\}$, and $N u \in$ $\operatorname{Im} L$, thus

$$
\Phi_{1}\left[\int_{0}^{t} f\left(s, a s^{\alpha-1}+b s^{\alpha-2}, a \Gamma(\alpha), a \Gamma(\alpha) s+b \Gamma(\alpha-1)\right) d s\right]=0
$$

and

$$
\Phi_{2}\left[\int_{0}^{t}(t-s) f\left(s, a s^{\alpha-1}+b s^{\alpha-2}, a \Gamma(\alpha), a \Gamma(\alpha) s+b \Gamma(\alpha-1)\right) d s\right]=0
$$

From (H8), we get $2 \Gamma(\alpha)|a|+\Gamma(\alpha-1)|b| \leq A$. Then, for $u \in \Omega_{2}$, we have

$$
\|u\|_{C^{\alpha-1}} \leq(2 \Gamma(\alpha)+1)|a|+(\Gamma(\alpha-1)+1)|b| \leq\left(1+\frac{1}{\Gamma(\alpha-1)}\right) A
$$

thus $\Omega_{2}$ is bounded. 
Next, for any $a_{1}, a_{2} \in \mathbb{R}$, define a linear isomorphism $J: \operatorname{Ker} L \rightarrow \operatorname{Im} Q$ by

$$
J\left(a_{1} t^{\alpha-1}+a_{2} t^{\alpha-2}\right)=\frac{2 a_{1} \Phi_{2}\left[t^{3}\right]-6 a_{2} \Phi_{1}\left[t^{2}\right]-\left(6 a_{1} \Phi_{2}\left[t^{2}\right]-12 a_{2} \Phi_{1}[t]\right) t}{2 \Phi_{1}[t] \Phi_{2}\left[t^{3}\right]-3 \Phi_{1}\left[t^{2}\right] \Phi_{2}\left[t^{2}\right]} .
$$

If (3.5) holds, set

$$
\Omega_{3}=\{u \in \operatorname{Ker} L: \lambda J u+(1-\lambda) Q N u=0, \lambda \in[0,1]\},
$$

where $Q$ is given by (2.7). For $u=a_{1} t^{\alpha-1}+a_{2} t^{\alpha-2} \in \Omega_{3}$, from $\lambda J u+(1-\lambda) Q N u=0$, we obtain

$$
\begin{aligned}
\Phi_{2}\left[t^{3}\right]\left(a_{1} \lambda+(1-\lambda) \Phi_{1}\left[\int_{0}^{t} N u(s) d s\right]\right) \\
-3 \Phi_{1}\left[t^{2}\right]\left(a_{2} \lambda+(1-\lambda) \Phi_{2}\left[\int_{0}^{t}(t-s) N u(s) d s\right]\right)=0
\end{aligned}
$$

and

$$
\begin{aligned}
& \Phi_{2}\left[t^{2}\right]\left(a_{1} \lambda+(1-\lambda) \Phi_{1}\left[\int_{0}^{t} N u(s) d s\right]\right) \\
& \quad-2 \Phi_{1}[t]\left(a_{2} \lambda+(1-\lambda) \Phi_{2}\left[\int_{0}^{t}(t-s) N u(s) d s\right]\right)=0 .
\end{aligned}
$$

By $2 \Phi_{1}[t] \Phi_{2}\left[t^{3}\right]-3 \Phi_{1}\left[t^{2}\right] \Phi_{2}\left[t^{2}\right] \neq 0$, it yields

$$
\left\{\begin{array}{l}
a_{1} \lambda+(1-\lambda) \Phi_{1}\left[\int_{0}^{t} N u(s) d s\right]=0 \\
a_{2} \lambda+(1-\lambda) \Phi_{2}\left[\int_{0}^{t}(t-s) N u(s) d s\right]=0
\end{array}\right.
$$

If $\lambda=1$, then $a_{1}=a_{2}=0$. Otherwise, if $\left|a_{1}\right|+\left|a_{2}\right|>B$, considering the above equalities and (3.5), we have

$$
\lambda\left(a_{1}^{2}+a_{2}^{2}\right)=-(1-\lambda)\left[a_{1} \Phi_{1}\left[\int_{0}^{t} N u(s) d s\right]+a_{2} \Phi_{2}\left[\int_{0}^{t}(t-s) N u(s) d s\right]\right]<0
$$

which contradicts $\lambda\left(a_{1}^{2}+a_{2}^{2}\right) \geq 0$. If (3.6) holds, then we take

$$
\Omega_{3}=\{u \in \operatorname{Ker} L:-\lambda J u+(1-\lambda) Q N u=0, \lambda \in[0,1]\}
$$

and, again, obtain a contradiction. Thus, in either case,

$$
\begin{aligned}
\|u\|_{C^{\alpha-1}} & =\left\|a_{1} t^{\alpha-1}+a_{2} t^{\alpha-2}\right\|_{C^{\alpha-1}} \\
& \leq\left|a_{1}\right|(1+2 \Gamma(\alpha))+\left|a_{2}\right|(1+\Gamma(\alpha-1)) \\
& \leq B(2+2 \Gamma(\alpha)+\Gamma(\alpha-1))
\end{aligned}
$$

for all $u \in \Omega_{3}$, that is, $\Omega_{3}$ is bounded. 
In the following, we shall prove that all the conditions of Theorem 2.1 are satisfied. Let $\Omega$ be a bounded open subset of $Y$ such that $\bigcup_{i=1}^{3} \overline{\Omega_{i}} \subset \Omega$. By Lemma 2.2 and standard arguments, we can prove that $K_{P}(I-Q) N: \Omega \rightarrow Y$ is compact, thus $N$ is $L$-compact on $\bar{\Omega}$. Then, by the above argument, we have

(i) $L u \neq \lambda N u$ for every $(u, \lambda) \in[(\operatorname{dom} L \backslash \operatorname{Ker} L) \cap \partial \Omega] \times(0,1)$,

(ii) $N u \notin \operatorname{Im} L$ for $u \in \operatorname{Ker} L \cap \partial \Omega$.

Finally, we will prove that (iii) of Theorem 2.1 is satisfied. Let $H(u, \lambda)= \pm \lambda J u+(1-\lambda) Q N u$. According to the above argument, we know

$$
H(u, \lambda) \neq 0 \quad \text { for } u \in \operatorname{Ker} L \cap \partial \Omega \text {. }
$$

Thus, by the homotopy property of degree, we have

$$
\begin{aligned}
\operatorname{deg}\left(\left.Q N\right|_{\operatorname{Ker} L}, \operatorname{Ker} L \cap \Omega, 0\right) & =\operatorname{deg}(H(\cdot, 0), \operatorname{Ker} L \cap \Omega, 0) \\
& =\operatorname{deg}(H(\cdot, 1), \operatorname{Ker} L \cap \Omega, 0) \\
& =\operatorname{deg}( \pm J, \operatorname{Ker} L \cap \Omega, 0) \neq 0 .
\end{aligned}
$$

Then, by Theorem 2.1, $L u=N u$ has at least one solution in $\operatorname{dom} L \cap \bar{\Omega}$, so that FBVP (1.1), (1.2) has a solution in $C^{\alpha-1}[0,1]$. The proof is complete.

\section{Competing interests}

The authors declare that they have no competing interests.

\section{Authors' contributions}

All authors contributed equally and significantly in this research work. All authors read and approved the final manuscript.

\section{Author details}

'Department of Statistics and Finance, Shandong University of Science and Technology, Qingdao, 266590, P.R. China.

2Department of Mathematics, Shandong University of Science and Technology, Qingdao, 266590, P.R. China.

\section{Acknowledgements}

The authors would like to thank the referees for carefully reading this article and making valuable comments and suggestions. This work is supported by the Foundation items: NSFC (10971179, 11071141), NSF (BS2010SF023, BS2012SF022) of Shandong Province.

Received: 11 January 2013 Accepted: 16 July 2013 Published: 7 August 2013

\section{References}

1. Podlubny, I: Fractional Differential Equations. Mathematics in Science and Engineering. Academic Press, New York (1999)

2. Kilbas, AA, Srivastava, HM, Trujillo, JJ: Theory and Applications of Fractional Differential Equations. North-Holland Mathematics Studies, vol. 204. Elsevier, Amsterdam (2006)

3. Babakhani, A, Gejji, VD: Existence of positive solutions of nonlinear fractional differential equations. J. Math. Anal. Appl. 278, 434-442 (2003)

4. Lakshmikantham, V, Leela, S, Devi, JV: Theory of Fractional Dynamic Systems. Cambridge Academic Publishers, Cambridge (2009)

5. Lakshmikantham, $\mathrm{V}$, Leela, S: Nagumo-type uniqueness result for fractional differential equations. Nonlinear Anal. TMA 71, 2886-2889 (2009)

6. Bai, Z: On positive solutions of a nonlocal fractional boundary value problem. Nonlinear Anal. TMA 72, 916-924 (2010)

7. Bai, Z, Lü, H: Positive solutions of boundary value problems of nonlinear fractional differential equation. J. Math. Anal. Appl. 311, 495-505 (2005)

8. Su, X: Boundary value problem for a coupled system of nonlinear fractional differential equations. Appl. Math. Lett. 22, 64-69 (2009)

9. Jiang, D, Yuan, C: The positive properties of the Green function for Dirichlet-type boundary value problems of nonlinear fractional differential equations and its application. Nonlinear Anal. TMA 72(2), 710-719 (2010)

10. Zhang, S: The existence of a positive solution for a nonlinear fractional differential equation. J. Math. Anal. Appl. 252, 804-812 (2000) 
11. Zhang, S: Existence of positive solution for some class of nonlinear fractional differential equations. J. Math. Anal. Appl. 278, 136-148 (2003)

12. Feng, $M, Z$ Zhang, $X, G e, W$ : New existence results for higher-order nonlinear fractional differential equation with integral boundary conditions. Bound. Value Probl. 2011, Article ID 720702 (2011)

13. Zhou, Y: Existence and uniqueness of solutions for a system of fractional differential equations. Fract. Calc. Appl. Anal. $12,195-204$ (2009)

14. Li, CF, Luo, XN, Zhou, Y: Existence of positive solutions of the boundary value problem for nonlinear fractional differential equations. Comput. Math. Appl. 59, 1363-1375 (2010)

15. Salem, HAH: On the nonlinear Hammerstein integral equations in Banach spaces and application to the boundary value problem of fractional order. Math. Comput. Model. 48, 1178-1190 (2008)

16. Ahmad, B, Nieto, Jj: Existence results for nonlinear boundary value problems of fractional integrodifferential equations with integral boundary conditions. Bound. Value Probl. 2009, Article ID 708576 (2009)

17. Cui, Y: Solvability of second-order boundary-value problems at resonance involving integral conditions. Electron. J. Differ. Equ. 2012, 45 (2012)

18. Zhang, Y, Bai, Z: Existence of solutions for nonlinear fractional three-point boundary value problems at resonance. J. Appl. Math. Comput. 36, 417-440 (2011)

19. Bai, Z, Zhang, Y: Solvability of fractional three-point boundary value problems with nonlinear growth. Appl. Math. Comput. 218, 1719-1725 (2011)

20. Zhao, Z, Liang, J: Existence of solutions to functional boundary-value problem of second-order nonlinear differential equation. J. Math. Anal. Appl. 373, 614-634 (2011)

21. Zhang, $X, F e n g, M, G e, W$ : Existence result of second-order differential equations with integral boundary conditions at resonance. J. Math. Anal. Appl. 353, 311-319 (2009)

22. Wang, G, Liu, W, Zhu, S, Zheng, T: Existence results for a coupled system of nonlinear fractional $2 m$-point boundary value problems at resonance. Adv. Differ. Equ. 2011, 44 (2011)

23. Webb, JRL: Remarks on non-local boundary value problems at resonance. Appl. Math. Comput. 216, 497-500 (2010)

24. Webb, JRL, Zima, M: Multiple positive solutions of resonance and nonresonance non-local fourth-order boundary value problems. Glasg. Math. J. 54, 225-240 (2012)

25. Hu, Z, Liu, W: Solvability for fractional order boundary value problem at resonance. Bound. Value Probl. 2011, 20 (2011)

26. Jiang, W: The existence of solutions for boundary value problems of fractional differential equations at resonance. Nonlinear Anal. 74, 1987-1994 (2011)

27. Mawhin, J: Topological degree and boundary value problems for nonlinear differential equations. In: Fitzpertrick, PM, Martelli, M, Mawhin, J, Nussbaum, R (eds.) Topological Methods for Ordinary Differential Equations. Lecture Notes in Mathematics, vol. 1537. Springer, Berlin (1991)

doi:10.1186/1687-1847-2013-233

Cite this article as: Zou and Cui: Existence results for a functional boundary value problem of fractional differential equations. Advances in Difference Equations 2013 2013:233.

\section{Submit your manuscript to a SpringerOpen ${ }^{\circ}$ journal and benefit from:}

- Convenient online submission

Rigorous peer review

- Immediate publication on acceptance

Open access: articles freely available online

- High visibility within the field

- Retaining the copyright to your article 